\title{
Gemeinwohl und öffentliches Wohneigentum
}

\author{
Direktdemokratische Initiativen zur Neuausrichtung öffentlicher \\ Wohnungsunternehmen in Frankfurt am Main und Berlin
}

\author{
Sebastian Botzem \\ Natalia Besedovsky
}

\begin{abstract}
Mietsteigerungen, Verdrängung und Verteilungskonflikte führen zu einer Repolitisierung der Wohnungspolitik, die auch öffentliche Wohnungsunternehmen betrifft. Soziale Bewegungen kritisieren vermehrt die Ausrichtung kommunaler und landeseigener Wohnungsgesellschaften und stellen den derzeitigen staatlichen Umgang mit öffentlichem Eigentum infrage. Wir verorten den Umgang mit öffentlichen Wohnungsunternehmen als Teil der Diskussion um eine gemeinwohlorientierte Wohnungspolitik und diskutieren Möglichkeiten der Neuausrichtung staatlicher Wohnungsgesellschaften. Anhand eines explorativen Fallvergleichs von Bürgerbegehren in Frankfurt am Main und Berlin untersucht dieser Artikel unterschiedliche Herangehensweisen und Forderungen direktdemokratischer Initiativen. Beide Initiativen waren erfolgreich darin, die Wirtschaftspraxis kommunaler Wohnungsunternehmen zum Gegenstand der öffentlichen Debatte zu machen, stießen allerdings auch auf Widerstände. Wir zeigen die Spielräume der Initiativen sowie die Grenzen direktdemokratischer Verfahren und diskutieren drei Dimensionen der Neuausrichtung öffentlicher Unternehmen: Bewirtschaftung, Mitbestimmung und Rechtsformänderung.
\end{abstract}

Ersteinreichung: 5. November 2020; Veröffentlichung online: 26. November 2021

An English abstract can be found at the end of the document.

\section{Einleitung: Repolitisierung der Wohnungsfrage}

Miet- und Wohnungsmärkte sind in vielen Städten seit Jahren angespannt: Die Preise für Neuvermietungen steigen schneller als Löhne und Einkommen. Wohnungsknappheit und hohe Mieten führen zur Verdrängung von Mieter_innen und zum Verlust von Wohnraum (Schönig/Kadi/Schipper 2017). Zugleich ist es zu einer Repolitisierung der Wohnungsfrage gekommen: Wohnungs- und Obdachlosigkeit sind verstärkt Gegenstand politischer Debatten, Bodenfragen beschäftigen die Kommunal-, Landes- und Bundespolitik. Auch auf Bundesebene ist der Mangel an bezahlbarem Wohnraum zum Politikum geworden. Eine Mietpreisbremse wurde eingeführt, die Bedingungen für Modernisierungen wurden modifiziert und gesetzliche Regelungen zum Milieuschutz präzisiert. Trotz verschiedener Versuche, dämpfend auf die Preisentwicklungen im Wohnungswesen einzuwirken und den Verlust von leistbarem Wohnraum zu stoppen, bleibt eine grundlegende Wende am Wohnungsmarkt bisher aus (Schönig/Vollmer 2021).

Der öffentliche Wohnungsbau in Deutschland stellte traditionell ein Korrektiv des renditeorientierten Wohnungsmarkts dar und trug wesentlich 
zur Wohnraumversorgung für Menschen aus niedrigen und mittleren Einkommensgruppen bei. Diesem politischen Auftrag wird er heute nicht mehr gerecht. Ab den 1990er Jahren kam es bundesweit zu Privatisierungen, insbesondere nach der Abschaffung der Wohngemeinnützigkeit 1989, die zuvor eine preisgedämpfte Bewirtschaftung des öffentlichen Wohnungsbestands geregelt hatte. Öffentliche Wohnungsbestände wurden vielfach an private Unternehmen veräußert und auf vermeintliche Kosteneffizienz getrimmt. Dennoch wird im politischen Diskurs vielfach daran festgehalten, dass Wohnen Teil der staatlichen Daseinsfürsorge sein soll, jedoch ohne dass ein grundsätzlicher Paradigmenwechsel weg von der neoliberalen Stadt- und Sozialpolitik erkennbar wäre.

Die Privatisierung und Finanzialisierung des Wohnungswesens, die in den vergangenen Jahren stattgefunden haben (Botzem/Dobusch 2012; Heeg 2013), verweisen auf das Spannungsverhältnis zwischen Wohnen als sozialem Recht auf der einen Seite und Immobilien als Anlageobjekten auf der anderen Seite. Andrej Holm (2011) konstatiert, dass das Mietverhältnis grundsätzlich die Spannung zwischen Gebrauchs- und Tauschwert des Wohnens verkörpere. Der Gebrauchswert von Wohnraum für Wohnende (die möglichst komfortabel, sicher und erschwinglich wohnen möchten) und der Tauschwert von Wohnraum für Eigentümer_innen (die einen möglichst hohen Mietzins oder einen hohen Wiederverkaufswert anstreben) stehen sich diametral gegenüber. Dieser Widerspruch ist durch verschiedene rechtliche Formen eingehegt und teilweise entschärft, etwa durch das Mietund Steuerrecht, bei Zwangsräumungen oder Eigenbedarfsregelungen. Wie sich das Widerspruchsverhältnis konkret gestaltet, ist somit Ergebnis politischer Kräfteverhältnisse (ebd.). Die stadtpolitischen Debatten um Gemeinwohl und gemeinwohlorientiertes Wohnen verweisen ebenfalls auf ein Spannungsverhältnis zwischen Partikularinteressen und allgemeinen Belangen (vgl. Schipper/Schönig 2021).

Ebenso facettenreich wie die Problemdiagnosen sind die Antworten auf die Wohnungskrisen. Im akademischen Raum wird über Möglichkeiten einer Abkehrvon neoliberalen Stadt(entwicklungs)politiken debattiert (Heeg 2013; Metzger/Schipper 2017; Schipper 2018; Schönig/Kadi/Schipper 2017; Rinn 2018; Sarnow 2019; Vogelpohl/Buchholz 2017; Vollmer 2019). Während die Mehrzahl der politischen Entscheidungsträger_innen weiterhin auf eine „Befriedung der Mittelschichten“(Vollmer 2019: 143) oder auf eine Strategie der „Verbürgerlichung“ (Rinn 2018: 9) setzt, streben zivilgesellschaftliche Bündnisse zum Teil grundlegende Veränderungen an, die bis hin zur Vergesellschaftung bestehenden Wohnraums reichen, wie es die Initiative „Deutsche Wohnen \& Co. enteignen“ in Berlin fordert.

Wir nehmen diese zivilgesellschaftliche Politisierung des Wohnens zum Anlass für eine Auseinandersetzung mit direktdemokratischen Initiativen zur Reform öffentlicher Wohnungsunternehmen, die sich in staatlichem Eigentum befinden. Im Mittelpunkt unseres Beitrags stehen Bürger_innenbegehren, mit deren Hilfe stadtpolitische Bündnisse anstreben, bindende Vorgaben für politische Entscheidungsträger_innen zu machen, um auf diese Weise öffentliche Unternehmen auf eine andere Wirtschaftsweise auszurichten. Konkret betrifft dies eine veränderte Bewirtschaftung des Bestands kommunaler Wohnungsunternehmen 
zugunsten einkommensschwacher Bewohner_innen sowie eine stärkere Kontrolle und Mitbestimmung in den Unternehmen.

Das Ziel dieses Beitrags ist es, anhand eines Fallvergleichs der beiden Bürger_innenbegehren „Mietentscheid Frankfurt“ und „Mietenvolksentscheid Berlin“, die wir als kritische Fälle für die Entwicklung einer Forschungsagenda interpretieren (Yin 2009), Merkmale zivilgesellschaftlicher Initiativen herauszuarbeiten, die eine Neuausrichtung staatlicher Wohnungsunternehmen fordern. Damit tragen wir zur Debatte um die Partizipation zivilgesellschaftlicher Gruppen in der Wohnungs- und Stadtpolitik bei, wobei unser Augenmerk auf Bürger_innen- und Volksbegehren als Mittel der Neuausrichtung öffentlicher Unternehmen liegt. Öffentliche Wohnungsunternehmen verstehen wir hier als - zumindest potenziell - sozial- und gesellschaftspolitische Gestaltungsressource, die die Möglichkeit eines gemeinwohlorientieren, planmäßigen Umgangs mit „Grundstücken und öffentlichen Wohnungsbeständen als Steuerungsinstrument“ (Holm 2013:52) beinhaltet. Entsprechend besteht unser Erkenntnisinteresse darin, zu analysieren, welche Forderungen direktdemokratische Initiativen für die Neuausrichtung öffentlicher Wohnungsgesellschaften entwickeln und inwieweit sie sich in verschiedenen Kontexten durchsetzen können. Unser Fokus liegt dabei auf den Schwierigkeiten aber auch den Chancen für direktdemokratische Initiativen, politische Forderungen an die Wohnungspolitik zu stellen, um konkrete Reformen einzuleiten.

Analytisch beziehen wir uns dabei auf den Begriff des Gemeinwohls. Dieser erfreut sich in der wissenschaftlichen Debatte, bei zivilgesellschaftlichen Akteur_innen wie auch bei politischen Entscheidungsträger_innen einer zunehmenden Verbreitung, bleibt aber unscharf und umstritten. Die Potenziale des Begriffs sehen wir in zweierlei Hinsicht: Zum einen nutzen wir den Begriff des Gemeinwohls zur Darstellung unterschiedlicher inhaltlicher und normativer Positionen der verschiedenen beteiligten Akteur_innen. Sowohl staatliche als auch zivilgesellschaftliche Akteur_innen legitimieren ihre Positionen (explizit oder implizit) mit einer Gemeinwohlorientierung, legen dabei allerdings unterschiedliche Verständnisse von Gemeinwohl zugrunde. Zum anderen ermöglicht der Begriff, Ungleichheiten in der Deutungsmacht unterschiedlicher Akteur_innen zu problematisieren, die sich insbesondere im Kontext der bisher dominierenden neoliberalen Wohnungspolitik ergeben.

Unser Artikel ist wie folgt aufgebaut: Zunächst erörtern wir den Begriff der Gemeinwohlorientierung im Wohnungswesen, skizzieren die Renditeorientierung am Wohnungsmarkt mit Blick auf öffentliche Unternehmen und beleuchten die Zunahme demokratischer Initiativen. Den Hauptteil bildet die vergleichende Fallanalyse direktdemokratischer Verfahren in Frankfurt am Main und Berlin, in der wir zunächst die methodischen Grundlagen skizzieren sowie die jeweiligen Kontexte der direktdemokratischen Verfahren in beiden Städten darstellen. Anschließend vergleichen wir die Entwicklungen, ordnen die Befunde ein und interpretieren sie in einem größeren wohnungspolitischen Kontext. 


\section{Gemeinwohlorientierung und öffentliches Wohnungswesen}

In gegenwärtigen stadtpolitischen Auseinandersetzungen wird vielfach auf das Ziel der Gemeinwohlorientierung Bezug genommen. Der Begriff erfreut sich großer Beliebtheit, obwohl - möglicherweise auch gerade weil - seine genaue Bestimmung unklar ist. Während die Klassiker der Philosophie immer wieder versuchten, Gemeinwohl zu definieren (für einen knappen Überblick vgl. Münkler/Fischer 1999; für eine ausführliche Diskussion vgl. Münkler/ Bluhm 2001), besteht in der gegenwärtigen sozialwissenschaftlichen Literatur weitestgehend Konsens, dass Gemeinwohl zwar normative Zielvorstellungen umfasst, aber kein konkret definiertes Konzept enthält oder enthalten kann. Dies betrifft sowohl die Frage der Gemeinschaft, also „wessen Wohl“ das Gemeinwohl ist (Offe 2019 [2001]), als auch die inhaltlichen Festlegungen, die es umfasst. In pluralistischen Demokratien gilt demnach, dass Gemeinwohl stets situations- und zeitgebunden ausgehandelt werden muss. Der situative und konflikthafte Charakter des Gemeinwohls erfordert daher eine begriffliche Offenheit, bringt jedoch auch die Gefahr eines „Catchall-Begriffs“ mit sich, unter dem sich viele Interessen versammeln können, ohne dass klar wäre, welche Bedeutungen ein- und ausgeschlossen sind. Folgt man Renate Mayntz, so kann Gemeinwohl weder wohlfahrtsökonomisch als Summe von Präferenzen der Gesellschaftsmitglieder aufgerechnet werden, noch kann es auf bloße Systemerhaltung reduziert werden (Mayntz 2001). Ein solches liberaldemokratisches Verständnis von Gemeinwohl ermöglicht also auch eine Distanzierung vom marktradikalen Paradigma des Neoliberalismus. Damit ist die Möglichkeit gegeben, mithilfe von Gemeinwohldiskursen gesellschaftliche Veränderungen zu begründen.

Ob und in welcher Form sich Konflikte um das Gemeinwohl entfalten, hängt allerdings von gesellschaftlichen Machtverhältnissen ab und ist damit auch eine empirische Frage. Häufig besteht hierbei ein strukturelles Machtgefälle, bei dem staatliche Akteur_innen auf der Basis formalisierter Macht für sich beanspruchen, im Sinne des Gemeinwohls zu agieren. Wurde im sozialwissenschaftlichen Diskurs der 2000er Jahre, etwa in Claus Offes Ausführungen, dieses Problem noch vornehmlich bei autokratischen Regimen gesehen, muss heute auch der neoliberale Staat dazugezählt werden (Sarnow 2019).

Durch die Krise der neoliberalen Wohnungspolitik (Vollmer/Kadi 2018) hat allerdings die Argumentation, dass Marktmechanismen zur effizientesten und daher wohlstandsmaximierenden Verteilung von Ressourcen (wie etwa Wohnraum) führen, an Legitimationskraft verloren. Es wird immer deutlicher, dass soziale Wohnungspolitik durch Privatisierung, Kommodifizierung und Allokation durch Marktmechanismen nicht zu realisieren ist (Heeg 2013). Trotzdem lässt sich, so der gegenwärtige Konsens in der kritischen Wohnungs- und Stadtforschung, keine Abkehrvon der neoliberalen hin zu einer postneoliberalen Wohnungspolitik erkennen (Rinn 2018; Vollmer/Kadi 2018). Ein normativ gehaltvoller Gemeinwohlbegriff, der auch soziale Ungleichheiten in den Fokus nimmt, muss also im Zweifelsfall gegen die im neoliberalen Diskurs ausgehandelte Definition vertreten werden. Zivilgesellschaftlichen Gruppen kommt daher bei der inhaltlichen Bestimmung eine besondere Bedeutung zu. 


\subsection{Gemeinwohl im Wohnungswesen}

Im Wohnungswesen gibt es verschiedene Akteur_innen, die sich für einen Gegenentwurf zur Immobilienwirtschaft und Wohnpolitik der letzten Jahrzehnte starkmachen. Dieser richtet sich gegen Gentrifizierung, Vermarktlichung und eine zunehmende Renditeorientierung des Wohnens und streitet für mehr Mitbestimmung. Zu den Merkmalen gemeinwohlorientierten Wohnens zählen nach diesem Verständnis neben bezahlbaren Mieten und barrierearmen Wohnungen auch gemeinschaftliche und solidarische Formen des Wohnens (BBSR 2019), die insbesondere in öffentlichen Unternehmen, in Genossenschaften, bei Stiftungen oder in selbstverwalteten Hausprojekten verortet werden. Gemeinwohl ist demnach „sozialintegrativ“ (Gennies 2021: 25), das heißt, dass ein Ausgleich zwischen den jeweiligen Einzel- und Gruppeninteressen geschaffen werden muss sowie darüber hinaus auch Belange des Umfelds und der Stadtgesellschaft Berücksichtigung finden.

Genossenschaften werden zum gemeinwohlorientierten Sektor gezählt. Ihre Grundprinzipien sind im Genossenschaftsgesetz festgehalten, wobei drei zentrale Merkmale gemeinschaftlichen Eigentums wichtig sind: Selbsthilfe (durch eine eigenständige Organisation), Selbstorganisation (durch gewählte Organe) und Selbstverwaltung (Genossenschaftsmitglieder sind/ wählen die Organmitglieder). Auf diese Weise wird dem Gedanken der Solidarität innerhalb einer Genossenschaft Rechnung getragen (Kuhnert/ Leps 2017: 42).

Breiter wird der Gemeinwohlbegriff bei Stiftungen ausgelegt. Formal geht es vor allem um eine steuerrechtliche Gemeinnützigkeit. Gemeinnützige Stiftungen nutzen das in ihnen gebündelte Kapital zum Erreichen der jeweiligen Stiftungszwecke, etwa für gemeinnützige Vorhaben wie Stadtentwicklung oder die Versorgung bestimmter Personengruppen. Traditionell fördern Stiftungen ausgewählte Bevölkerungsgruppen, wie beispielsweise ältere Personen oder Menschen mit Mobilitätseinschränkungen, indem sie ihnen preisgünstigen und bedarfsgerechten Wohnraum zur Verfügung stellen. Dies gilt beispielsweise für kirchliche und soziale Träger, die auf diese Weise Notlagen auffangen. Andere Stiftungen werden direkt im Immobilienund Grundstücksmarkt aktiv. Sie setzen ihr Vermögen ein, um „Grund und Boden aus dem ,spekulativen Verwertungskreislauf herauszulösen“ (BBSR 2019: 34).

Auch privatrechtlich verfasste solidarische Hausprojekte, wie sie beispielsweise im Netzwerk des Mietshäusersyndikats versammelt sind, verstehen sich als Gegenentwürfe zur renditeorientierten Wohnungswirtschaft. Das Mietshäusersyndikat etwa fungiert als Dach- und Beratungsorganisation, die einzelne Hausprojekte unterstützt und durch eine rechtliche Verschachtelung verhindert, dass einzelne Gebäude oder Grundstücke weiterverkauft werden können (BBSR 2019: 50; Schipper 2018). Ein weiteres Beispiel selbstverwalteter Wohnprojekte mit stadtpolitischem Anspruch und gemeinwohlorientierter Ausrichtung sind Community Land Trusts (CLT). Diese setzen auf Selbstverwaltung und dauerhafte Partizipation nachbarschaftlicher Akteur_innen. Pächter_innen und Nutzer_innen können stimmberechtigte Mitglieder sein, die auch formal an Entscheidungsprozessen beteiligt sind (Horlitz 2019: $108 \mathrm{f}$.). 
All diesen Organisationsformen ist gemein, dass sie sich den Marktmechanismen und der Renditeorientierung zumindest zum Teil zu entziehen versuchen und sich als Gegenentwürfe zu profitmaximierenden Unternehmen verstehen. Die formale rechtliche Kategorisierung als gemeinnützig agierende Organisation ist dabei verhältnismäßig eindeutig, die tatsächliche Einschätzung der jeweiligen Gemeinwohlorientierung ist dagegen anspruchsvoll. Denn es könnte kritisch argumentiert werden, sie priorisierten statt einer Gemeinwohlorientierung auf gesellschaftlicher Ebene die Partikularinteressen ihrer Mitglieder. Hieran werden die Zielkonflikte deutlich, denen gemeinwohlorientierte Organisationen ausgesetzt sind. Dies betrifft insbesondere den Konflikt zwischen der Erfüllung der von außen herangetragenen Erwartungen an ihre Gemeinwohlorientierung einerseits und dem organisationalen Eigeninteresse andererseits. Letzteres umfasst sowohl die Interessen der einzelnen Mitglieder als auch das Gesamtinteresse der Organisation (etwa das Überleben oder die Handlungsfähigkeit der Organisation) (Mayntz 1992). Nichtmitglieder werden in diesen Fällen als Anspruchsberechtigte vom Gemeininteresse ausgeschlossen.

Auch vor dem Hintergrund dieses Zielkonflikts und der stärkeren Verbreitung von Organisationen, die eine Renditemaximierung ablehnen, lässt sich eine Politisierung des Gemeinwohlkonzepts seitens zivilgesellschaftlicher Initiativen beobachten. Vielfach beziehen sich „Recht-auf-StadtBewegungen" auf Gemeinwohlorientierung als Aspekt der Stadtpolitik (Gestring/Ruhne/Wehrheim 2014; Holm 2014; Vollmer 2019). Derzeit wird diskutiert, inwieweit der Gemeinwohlbegriff als politischer Begriff hilfreich sein könnte (Vollmer 2021). Trotz der oben genannten Probleme wird der Vorteil des Gemeinwohlbegriffs darin gesehen, dass er nur schwer ins Begriffsrepertoire des Marktliberalismus passt (ebd.). Damit stellt bereits die Verwendung des Begriffs eine Verschiebung des Diskurses dar, indem sie eine Distanzierung vom neoliberalen Paradigma bei der Wohnraumversorgung erlaubt. Um über eine bloße Kritik an der neoliberalen Stadt hinauszugelangen, ist es jedoch für soziale Bewegungen notwendig, einen inhaltlich ausgearbeiteten Gemeinwohlbegriff zu entwickeln (ebd.). Die Debatte um Gemeinwohl dokumentiert damit auch den Anspruch zivilgesellschaftlicher Akteur_innen, gleichberechtigt an stadtpolitischen Diskursen teilzunehmen. „So streiten zum Beispiel soziale Bewegungen mit staatlichen Institutionen oder privatwirtschaftlichen Vertreter_innen darum, was das Gemeinwohl ist und wie es zu erreichen sei." (Vollmer 2019: 2) Dabei organisieren und vernetzen sie sich und kooperieren auch mit anderen Initiativen. So kommen Gruppen mit unterschiedlicher politischer Ausrichtung zusammen und können gesamtstädtische Fragen auf die Agenda setzen (Holm 2014).

Ein hilfreicher Bezugspunkt für eine substanzielle Füllung des Gemeinwohlbegriffs ist die Diskussion um die Etablierung einer Neuen Wohnungsgemeinnützigkeit, die 2015 von zwei Studien für die Bundestagsfraktionen von DIE LINKE und Bündnis 90/Die Grünen angestoßen wurde (Holm/ Horlitz/Jensen 2015; Kuhnert/Leps 2017). Die Grundidee stellt im Kern einen Rückbezug zur Wohnungsgemeinnützigkeit in der alten Bundesrepublik dar, die zum 1. Januar 1990 abgeschafft wurde. Dort war festgelegt, „die Verantwortung für eine soziale Wohnungsversorgung auf der Ebene der 
Unternehmen selbst festzuschreiben und durch steuerliche Entlastungen den gesellschaftlich gewünschten und notwendigen - also den gemeinnützigen Zweck einer dauerhaften sozialen Wohnungsversorgung zu fördern“ (Holm/ Horlitz/Jensen 2017: 22). Die Wohnungsgemeinnützigkeit beinhaltete die steuerliche Förderung von - privaten wie öffentlichen - Wohnungsmarktakteur_innen, damit diese preisgünstigen Wohnraum bereitstellen konnten. Sie war durch vier Merkmale gekennzeichnet (Kuhnert/Leps 2017): Beschränkung der Aktivitäten gemeinnütziger Wohnungsunternehmen auf Bau, Verwaltung und Verkauf von Wohnungen, Kostendeckelung bei Wohnungsdienstleistungen, Rückführung nicht ausgezahlter Überschüsse in das Unternehmen sowie Gewinnbeschränkung von Genossenschaftsmitgliedern und Eigentümer_innen gemeinnütziger Wohnungsgesellschaften auf maximal vier Prozent der Kapitaleinlage pro Jahr.

Eine Wiedereinführung der Gemeinnützigkeit brächte Steuervorteile und Förderungen entsprechender Organisationen mit sich, ist aber zugleich an Bedingungen geknüpft (ebd.: 22-28). Diese umfassen beispielsweise Gewinnbeschränkungen und eine Zweckbindung der Einnahmen. Außerdem sollen, unter anderem durch einkommensabhängige Mieten, leistbare Wohnungen bereitgestellt werden, die bevorzugt an niedrige Einkommensgruppen sowie am Wohnungsmarkt diskriminierte Wohnungssuchende vermietet werden. Darüber hinaus beinhaltet die Neue Wohnungsgemeinnützigkeit Möglichkeiten der Mietermitbestimmung und erweiterte Transparenzgebote (vgl. ebd.: 320 ff.). Die Neue Wohnungsgemeinnützigkeit zielt zwar nicht nur auf öffentliche Unternehmen, diese spielen aber wegen der Anzahl von Wohnungen, die sie bewirtschaften, eine herausgehobene Rolle für einen möglichen Wandel der Wohnungspolitik. Die Bedeutung öffentlicher Wohnungsunternehmen unterstreicht der Begriff von Wohnen bzw. Wohnraum als „sozialer Infrastruktur“ (vgl. Holm 2013; Jensen 2021). In Anlehnung an die (Re-)Kommunalisierung öffentlicher Dienstleistungen in den Bereichen Wasser- und Stromversorgung betont der Infrastrukturbegriff die Bedeutung öffentlichen Eigentums und unterstreicht das Bemühen von Kommunen, „Handlungs- und Gestaltungsmacht über die Infrastrukturen und ihre Bereitstellung zurückzugewinnen“"(Jensen 2021: 151).

\subsection{Renditeorientierung und öffentlicher Wohnungsbestand}

Öffentliche Wohnungsunternehmen verfügen in Deutschland über etwa 2,8 Millionen Wohnungen und damit über circa zwölf Prozent des Gesamtbestands der vermieteten Wohnungen (vgl. Hunger 2017: 158). Der Bestand hat in den zurückliegenden Jahrzehnten allerdings aufgrund von Privatisierungen stark abgenommen. Auch steuerrechtliche Änderungen haben die Handlungsbedingungen des öffentlichen Wohnungssektors eingeschränkt. Besonders einschneidend war die Abschaffung der oben genannten Wohnungsgemeinnützigkeit. Sie wurde vollzogen auf Druck der Immobilienwirtschaft, die sich der Angebotskonkurrenz des gewinnkontrollierten Wohnungssektors entledigen und die Märkte für die gewerbliche Bauwirtschaft, für Immobilienentwickler_innen und Immobilienverwaltungsgesellschaften öffnen wollte (Mertens 2015). Davon waren rund 1.800 gemeinnützige Wohnungsunternehmen betroffen. 
Ab den 1990er Jahren kam es zu weitreichenden Veränderungen auf dem Wohnungsmarkt. Nach der deutschen Einheit setzten sich anhaltende Privatisierungs- und Liberalisierungsprozesse durch, die insbesondere den Einfluss internationalisierter Finanzmarktakteur_innen beförderten (ebd.; Metzger 2020). Zudem verkauften große Industrieunternehmen ihre Werkswohnungen (Unger 2018) und es kam zur Privatisierung großer Wohnungsbestände durch Bund, Länder und Kommunen. Zwischen 1999 und 2011 wurden in Deutschland insgesamt 549.000 öffentliche Wohneinheiten an private Eigentümer_innen verkauft (Held 2011: 675). Besonders auffällig waren die Aktivitäten in Berlin, Kiel und Dresden, wo ein großer Teil der kommunalen Wohnungen veräußert wurde (ebd.). Auf diese Weise wurden zwar einmalige Einnahmen generiert, die öffentliche Wohnraumversorgung wurde jedoch aufgegeben. Auch die Aktivitäten der andernorts verbliebenen öffentlichen Wohnungsunternehmen wurden stärker an Profiterwartungen ausgerichtet (vgl. Krüger 2019), teils basierend auf der Annahme, eine marktorientierte Bewirtschaftung würde zu einem effizienteren Ressourceneinsatz führen, teils aber auch, um auf Kosten der Bewohner_innen die öffentliche Finanzausstattung zu verbessern.

Vor allem nach der Jahrtausendwendestieg der Einfluss renditeorientierter Immobilieninvestoren aus dem In- und Ausland auf den Wohnungsmarkt stark an. Dies hat eine Finanzialisierung des Wohnens zur Folge, die zu Verdrängung und Gentrifizierung führt (Heeg 2013). Finanzialisierung umfasst die Dominanz von Profitinteressen und die Privilegierung von Investor_innen gegenüber den Bedürfnissen von Mieter_innen (Botzem/ Dobusch 2012). Manuel Aalbers interpretiert diese Veränderungen als neue wohnungspolitische Etappe und schlussfolgert, dass das Wohnungswesen in ein postfordistisches, neoliberales und finanzialisiertes Regime eingetreten sei, in dem sowohl hypothekarisch belastetes Wohneigentum als auch subventionierter Mietwohnungsbau vor allem dem Zweck dienten, die Renditeerwartungen der Finanzmärkte zu bedienen (Aalbers 2017: 543). Neoliberale Verwertungslogiken des Wohnens zeigen sich darüber hinaus auch in der Stadtplanung (Kamleithner 2009), im sozialen Wohnungsbau (Schönig 2018) oder bei Auseinandersetzungen um den Umgang mit Grünflächen (Hilbrandt 2019).

Privatisierung und Finanzialisierung des Wohnungsmarkts haben die Rahmenbedingungen für kommunale Wohnungsgesellschaften stark verändert. Diese konkurrieren mit kommerziellen Anbietern um zunehmend teurer werdende Böden und Immobilien, wenn sie ihren Wohnungsbestand erweitern wollen. Kommunen geben ein wichtiges Steuerungsinstrument für eine gemeinwohlorientierte Stadtpolitik aus der Hand, wenn sie nicht mehr direkt in Form eigener Wohnraumbewirtschaftung auf das Marktgeschehen Einfluss nehmen können (vgl. Holm 2011). Inzwischen wird die Rolle öffentlicher Unternehmen wieder stärker diskutiert und gefordert, den öffentlichen Wohnungsbestand auszubauen und benachteiligte Gruppen in den Mittelpunkt der öffentlichen Wohnraumversorgung zu stellen. Allerdings hängt das Engagement öffentlicher Unternehmen davon ab, welche Vorgaben ihre kommunalen Eigentümer_innen ihnen machen. Dass der zivilgesellschaftliche Unmut über mangelnde soziale Vorgaben der Gesellschafter gegenüber den Unternehmen zunimmt, zeigt sich auch an der 
Verbreitung direktdemokratischer Verfahren, in denen mehr politische Steuerung eingefordert wird.

\subsection{Direktdemokratische Initiativen zur Steuerung kommunaler Wohnungsunternehmen}

Direktdemokratische Verfahren der Bürger_innenbeteiligung, insbesondere Bürger_innen- und Volksbegehren, nehmen seit den 1990er Jahren stark zu (Mittendorf/Schiller 2008). „Mit diesen Verfahren kann eine bestimmte Anzahl von Bürgern einen Gesetzes- oder Entscheidungsvorschlag präsentieren und eine Volksabstimmung herbeiführen, falls nicht das jeweilige Parlament den Vorschlag übernimmt oder dem Anliegen sonst wie abhilft“ (ebd.: 142). Solche direktdemokratischen Verfahren oder Plebiszite beziehen sich auf konkrete Sachentscheidungen, unterliegen aber formalen Einschränkungen. So wird die Zulässigkeit von Anträgen von der Verwaltung überprüft, es sind Fristen zu beachten und zudem Quoren der Beteiligung erforderlich, sowohl bei der Einleitung von Bürger_innenbegehren (Unterstützungsunterschriften) als auch bei einer folgenden Abstimmung (Zustimmungsquorum als Anteil aller Stimmberechtigten). Nicht selten legen öffentliche Institutionen die rechtlichen Kriterien streng aus und erhöhen damit die praktischen Hürden in Beteiligungsverfahren. Da Einleitung und Durchführung eines Bürger_innen-bzw. Volksbegehrens anspruchs- und voraussetzungsvoll sind, stehen hinter den formal verantwortlichen Gruppen und Personen breite, oft stadtweite Netzwerke aus Initiativen und politischen Interessengruppen. Die Sammlung von Unterschriften ist der Ausgangspunkt eines Begehrens, mit dessen Hilfe eine Mobilisierung erfolgt und politischer Druck entfaltet werden kann. Kommen in dieser ersten Stufe die notwendigen Stimmen zusammen, wird der vorgeschlagene Textentwurf entweder der gesamten Bevölkerung zur Abstimmung vorgelegt (wie in Frankfurt am Main; siehe 3.1), oder es ist eine weitere Unterschriftensammlung erforderlich, bevor eine Volksabstimmung durchgeführt werden kann (wie in Berlin; siehe 3.2).[1]

In den zurückliegenden Jahren sind kommunale Wohnungsunternehmen und ihre Bewirtschaftungspraxis vermehrt Gegenstand direktdemokratischer Verfahren geworden. Zahlreiche lokale Initiativen und stadtpolitische Bündnisse strengen Bürger_innen- oder Volksbegehren an, um Veränderungen bei öffentlichen Unternehmen herbeizuführen. Dabei werden Forderungen erhoben, die auf eine wirtschaftliche und/oder organisatorische Neuausrichtung bestehender öffentlicher Wohnungsunternehmen abzielen, oder es werden (Neu-)Gründungen kommunaler Wohnungsgesellschaften angestrebt, die eine wichtige Rolle bei der sozialen Wohnraumversorgung einnehmen sollen. Somit sind Bürger_innenbegehren als direkte Kritik am bisherigen - in der Regel stark an der profitorientierten Immobilienwirtschaft ausgerichteten - Wirtschaften öffentlicher Wohnungsunternehmen zu verstehen. Es geht also nicht darum, die Eigentumsstrukturen zu verändern - es handelt sich ja bereits um Unternehmen, deren Gesellschafter die öffentliche Hand ist. Die Forderungen zielen vielmehr darauf ab, eine andere Form der Bewirtschaftung sowie Mitbestimmung und strengere Kontrollmöglichkeiten durchzusetzen. 
Mittlerweile lässt sich ein bundesweiter Trend beobachten: Eine Übersicht allervom Verein „Mehr Demokratie“erfassten Bürger_innenbegehren enthält allein für das Jahr 2019 vier Initiativen, die unmittelbar auf die Unternehmensentscheidungen öffentlicher Wohnungsgesellschaften ausgerichtet sind (Stuttgart: für bezahlbaren Wohnraum: Mietentscheid Stuttgart; Frankfurt am Main: für eine feste Quote beim sozialen Wohnungsbau: Mietentscheid Frankfurt; Jena: für mehr sozialen Wohnungsbau: Rückkauf von Anteilen an der Jenawohnen $\mathrm{GmbH}$, mehr Mitbestimmungsrechte für Mieter_innen; Osnabrück: für die Gründung einer kommunalen Wohnungsbaugesellschaft) (Mehr Demokratie 2021). Die Vorhaben beinhalten konkrete Maßnahmen, die sich direkt auf die Geschäftspraxis kommunaler Wohnungsgesellschaften beziehen: Verkaufsstopp für öffentliche Grundstücke; vermehrter Neubau; verstärkte, zum Teil sogar ausschließliche Schaffung von öffentlich gefördertem Wohnraum; Deckelung oder Reduzierung von Mietkosten.

Auch auf Ebene der Bundesländer finden sich vermehrt Initiativen, die wohnungspolitische Forderungen mithilfe von Volksbegehren durchsetzen wollen: In Schleswig-Holstein gibt es Bemühungen, per Volksinitiative die Landesverfassung zu ändern und Förderung, Schaffung und Erhalt von bezahlbarem Wohnraum als Ziele in die Verfassung aufzunehmen. In Hamburg strebt die Volksinitiative „Boden und Wohnraum behalten - Hamburg sozial gestalten!“ an, verbindlich festzulegen, dass die Stadt Hamburg keine öffentlichen Liegenschaften mehr verkauft. In Berlin zielt das Volksbegehren „Deutsche Wohnen \& Co. enteignen“ darauf, die Vergesellschaftung der Berliner Bestände großer Immobilienkonzerne herbeizuführen, die über 3.00o Wohnungen vermieten. In Bayern hat die Initiative „6-Jahre-Mietenstopp“ im Zuge eines Volksbegehrens 52.00o Unterschriften gesammelt, um durchzusetzen, dass in 162 bayerischen Städten und Gemeinden die Mieten sechs Jahre lang eingefroren werden.

Stadtpolitische Fragen spielen traditionell eine große Rolle bei Bürger_innenbegehren. Dazu gehören die Bauleitplanung, die Verkehrsplanung und insbesondere die Förderung des Radverkehrs (Mehr Demokratie 2020). Bürger_innenbegehren bringen jedoch vielfältige Interessen zum Ausdruck, die sich nicht immer eindeutig in das politische Spektrum einordnen lassen. Neben Initiativen für zusätzlichen Wohnungsbau finden sich beispielsweise etliche Bemühungen gegen den Ausbau der Windenergie, gegen Mobilfunkmasten oder gegen die Ausweitung von Neubau- und Industriegebieten (Mehr Demokratie 2021).

\section{Fallvergleich: Wohnungspolitiken in Frankfurt am Main und Berlin}

Die vorliegende Studie basiert auf einem qualitativen Fallvergleich im Sinne eines explorativen Paarvergleichs (vgl. Mayring 2002) der Entwicklungen um den Mietentscheid in Frankfurt am Main und das Mietenvolksbegehren in Berlin. Neben einer detaillierten Fallbeschreibung dient der Fallvergleich der Einordnung von partizipativen Initiativen im Bereich der Wohnungspolitik sowie der Systematisierung der Ziele, Bedingungen und Vorgehensweisen einer demokratischen Steuerung öffentlicher Wohnungsgesellschaften. Der Fallvergleich ermöglicht es zudem, Faktoren herauszuarbeiten, die über 
den Ausgang und die Konsequenzen solcher Initiativen entscheiden. Das Ziel ist dabei, weitergehende forschungsleitende Annahmen zu den Möglichkeiten und Grenzen der Steuerung und Demokratisierung öffentlicher Wohnungsunternehmen zu generieren (Döring/Bortz 2016).

Da die Rolle zivilgesellschaftlicher Initiativen in beiden Städten und deren Beitrag zur Wohnungspolitik bereits häufig Forschungsgegenstand waren, stützt sich die Analyse zum einen auf die bereits umfangreich vorliegenden Befunde (vgl. insb. Vollmer 2019; Vogelpohl et al. 2017; Schipper 2018; Metzger/Schipper 2017; Lebuhn 2008; Künkel 2018; Diesselhorst 2018). Zusätzlich zu dieser sekundäranalytischen Betrachtung werden weitere Quellen herangezogen: Primärquellen der Wohnungsmarktakteur_innen, Stellungnahmen, Presseberichte, Verwaltungsdokumente und Statements zivilgesellschaftlicher Akteur_innen sowie ein vertiefendes Hintergrundgespräch.

Für den Paarvergleich wurden Frankfurt am Main und Berlin gewählt, da sowohl die Initiativen selbst als auch ihre jeweiligen Erfolge beziehungsweise Erfolgsaussichten deutliche Unterschiede aufweisen. Zugleich gibt es einige Gemeinsamkeiten, die einen Vergleich möglich machen: Beide Großstädte haben angespannte Wohnungsmärkte mit hohen Preissteigerungen, weisen zugleich aber eine lange Tradition wohnungs- und stadtpolitischer Initiativen auf. Zudem zeichnet die beiden untersuchten zivilgesellschaftlichen Initiativen aus, dass sie nicht nur auf bestimmte Einzelentscheidungen der Stadtverwaltung Einfluss nehmen, sondern einen grundlegenden Wandel der Bewirtschaftung öffentlicher Wohnungsbestände herbeiführen wollen. Sie haben dauerhafte sozialpolitische Ziele, die den grundlegenden Charakter der Wohnungsunternehmen berühren. In beiden Fällen sehen die Initiator_ innen der Begehren eine Abkehr von der profitorientierten Bewirtschaftung des Wohnungsbestands vor. In Berlin wird darüber hinaus eine Änderung der Rechtsform und eine Beteiligung der Mieter_innenschaft in den Kontrollgremien gefordert. Trotz unterschiedlicher ökonomischer Rahmenbedingungen kommt beiden Städten eine Vorreiterrolle zu. Aufgrund ihrer weitreichenden Forderungen und stadtpolitischen Bedeutung interpretieren wir die Fälle in Frankfurt am Main und Berlin als kritische Fälle im Sinne von Yin (2009), also als Fälle, aus deren Bedeutung sich die Möglichkeit verallgemeinerbarer Schlussfolgerungen ergibt.

Im Folgenden stellen wir zunächst die Entwicklungen in Frankfurt am Main vor, auch wenn das formale Bürger_innenbegehren dort erst im Jahr 2018 und damit nach dem Berliner Mietenvolksentscheid angestrengt wurde. Die derzeit laufende Initiative „Mietentscheid Frankfurt“ ist relativ eng gefasst und zielt darauf ab, die Ausrichtung der Geschäftspolitik der stadteigenen Wohnungsgesellschaft ABG Frankfurt grundlegend zu verändern. Anschließend untersuchen wir die Aktivitäten rund um den „Mietenvolksentscheid Berlin“, der 2015 begonnen wurde und neben der Wohnraumbewirtschaftung auch die Rechtsform der Unternehmen verändern und eine Mieter_innenmitbestimmung als Teil der Neuausrichtung der städtischen Wohnungsunternehmen durchsetzen wollte.

Trotz historischer, politischer und wirtschaftlicher Eigenheiten weisen Frankfurt am Main und Berlin zahlreiche Gemeinsamkeiten auf. Dazu gehören die überdurchschnittliche Mietpreisentwicklung, die große Bedeutung 


\begin{tabular}{lcc}
\hline & Frankfurt/Main & Berlin \\
\hline Bevölkerung 2018 & 746.878 & 3.644 .826 \\
Anstieg in zehn Jahren (2008 bis 2018) & $+13 \%$ & $+6,2 \%$ \\
\hline Anteil fremdgenutztes Wohneigentum (2018) & $81 \%$ & $82 \%$ \\
\hline Wohnungsbestand 2018 insgesamt & 398.200 & 1.949 .300 \\
Wohnungen im Besitz kommunaler Gesellschaften (2018) & 53.000 & 306.000 \\
Anteil kommunaler Wohnungen am Gesamtbestand & $13,3 \%$ & $15,7 \%$ \\
\hline
\end{tabular}

kommunaler Wohnungsgesellschaften und eng vernetzte stadtpolitische Bewegungen. Tabelle 1 verdeutlicht die Parallelen. In zweierlei Hinsicht variieren die Städte allerdings: Frankfurt am Main weist ein höheres durchschnittliches Haushaltseinkommen auf als Berlin und ist zugleich stärker von konservativen politischen Mehrheiten geprägt.

Beide Städte sind in den zurückliegenden Jahren stark gewachsen und ähneln sich hinsichtlich der Wohnraumversorgung: Weniger als 2o Prozent aller Wohnungen werden von ihren Eigentümer_innen bewohnt, vier Fünftel aller Wohnungen sind also vermietet. Für Mieter_innen mit Bestandsverträgen ergeben sich in laufenden Verträgen meist relativ moderate Mietsteigerungen. Bei Neu- und Wiedervermietungen zeigen sich dagegen drastische Preissteigerungen. Von 2012 bis 2019 sind die durchschnittlichen Quadratmeterpreise für wiedervermietete Wohnungen in Frankfurt am Main von 10,42 auf 14,21 €/qm nettokalt gestiegen (plus 36 Prozent), in Berlin sogar um 65 Prozent - von 7,0 auf 11,50 €/qm nettokalt (Deutscher Bundestag 2020).

Frankfurt am Main ist die größte Stadt des Bundeslandes Hessen und Oberzentrum der Rhein-Main-Region, während Berlin als Hauptstadt deutlich größer ist und ein eigenes Bundesland darstellt.[2] Daher sind Bürger_innenbegehren in Frankfurt am Main darauf ausgerichtet, einen Bürger_innenentscheid als Abstimmung zu einer kommunalpolitischen Sachfrage durchzuführen. In Berlin zielen Volksbegehren auf die Durchführung eines Volksentscheids, der eine Abstimmung über einen Gesetzentwurf oder über einen anderen Beschlussentwurf auf Landesebene umfasst. Beide Städte zeichnen sich durch eine kritische Stadtgesellschaft aus, die auf eingespielte Routinen zivilgesellschaftlichen Widerstands zurückgreifen kann, wenn auch die Frankfurter Stadtpolitik stärker von bürgerlich-konservativen Parteien geprägt ist als diejenige in Berlin.

\subsection{Frankfurt am Main: \\ Soziale Vermietungspolitik des städtischen Unternehmens $A B G$}

Frankfurt am Main ist einer der teuersten Orte Deutschlands, obwohl es eine große städtische Wohnungsgesellschaft gibt. Die Geschichte der ABG Frankfurt Holding reicht zurück bis in das Jahr 1890, in dem sie als Aktienbaugesellschaft für kleine Wohnungen (ABG) gegründet wurde. Zunächst ein philanthropisches Projekt engagierter Einzelpersonen, übernahm die Stadt in den 1920er Jahren die ABG. Nach dem Zweiten Weltkrieg wurde die Hälfte der 11.00o beschädigten oder zerstörten Wohnungen wiederaufgebaut, zudem wurden weitere Wohnungen gebaut. So besaß die ABG im Jahr 1990
Tab. 1 Städtevergleich

Frankfurt am

Main und Berlin

(Quelle: eigene Zusammenstellung) 
circa 20.00o Wohnungen. Später wurde sie zum Kernstück einer fusionierten Wohnungs- und Immobiliengesellschaft, der ABG Frankfurt Holding $\mathrm{GmbH}$. Die neue Holdinggesellschaft ist für einen Bestand von über 50.000 Wohnungen verantwortlich und befindet sich vollständig im Besitz der Stadt. 25 Prozent aller Frankfurter Bürger_innen leben in Wohnungen der ABG (ABG 2016). Die ABG betreibt, verwaltet und baut Wohnraum und ist einer der großen wohnungspolitischen Akteur_innen in Frankfurt am Main. Obwohl sich die Holding in städtischem Eigentum befindet, ähnelt ihre Bewirtschaftungspraxis der profitgetriebener privater Wohnungsunternehmen (Schipper 2016). Das Unternehmen bewirtschaftet eigene und fremde Wohnungen, betreibt eine Reihe von Tochtergesellschaften und führt jährlich zwischen fünf und acht Millionen Euro an den städtischen Haushalt ab.

Kritik an der Wohnungsmarktsituation und der Rolle der ABG formierte sich bereits in den frühen 2010er Jahren, auch mit Blick auf die Gewinnorientierung der ABG (Metzger/Schipper 2017). Zwei zivilgesellschaftliche Netzwerke wurden zum Rückgrat einer stadtweiten Bewegung: 2011 wurde „Wem gehört die Stadt?“ gegründet, ein unabhängiges Netzwerk von Stadtaktivist_innen, ein Jahr später das „Aktionsbündnis für bezahlbaren Wohnraum" mit Verbindungen zu den Gewerkschaften, zur SPD und zur LINKEN. Beide Netzwerke waren zunächst erfolgreich in der Mobilisierung, verloren aber ab 2013 wieder an Dynamik (Schipper 2018: 74).

Nach Kritik an der Profitorientierung der ABG gab es 2014 leichte Korrekturen der Unternehmensstrategie. Die konservativ-grüne Ratsmehrheit beschloss, die anstehenden Mieterhöhungen für einen Zeitraum von drei Jahren auf 15 Prozent zu begrenzen (während die Bundesbestimmungen damals noch 20 Prozent zuließen). Diese geringfügige Abweichung gegenüber dem gesetzlich zulässigen Maximum wurde von der Regierungskoalition als Beleg für eine ausreichende soziale Ausrichtung der ABG gelobt und von der konservativ-grünen Ratsmehrheit wie folgt gerechtfertigt (STVV 2014):

- Eine Reihe von Mieter_innen der ABG sei ausreichend wohlhabend und benötige keine unterdurchschnittliche Mieterhöhung.

- Die Gewinne der ABG gingen an die Stadt als Ganzes und stünden daher allen Bürger_innen zur Verfügung, während ermäßigte Mieten nur denjenigen zugutekämen, die in den Wohnungen leben.

- Die Stadt benötige ein gewinnorientiertes Unternehmen, um öffentlichen Wohnungsbau zu betreiben und zukünftig mehr investieren zu können.

Die Argumente von 2014 verdeutlichen die Ambivalenz des Gemeinwohlbegriffs. In den Augen der Ratsmehrheit agiert die ABG schon dann im Sinne einer sozial ausgerichteten Wohnungspolitik, wenn sie sich auf immobilienwirtschaftliche Marktdynamiken einlässt. Dabei wird die ABG als wohnungswirtschaftliches Instrument angesehen, das auf dem finanzialisierten Wohnungsmarkt entsprechend der vorherrschenden Profitlogik agieren soll. Die Profitorientierung und die Ausschüttung der Gewinne an die Stadt werden zudem zur Legitimierung der finanzialisierten Wohnungspolitik als Gemeinwohlorientierung genutzt, während der Schutz der Mieter_innen als Bedienung von Partikularinteressen gedeutet wird. Dabei wird die politische Umkämpftheit des Gemeinwohls deutlich: Die Initiative, die auf eine Neuausrichtung der ABG hinwirkt, sieht im Gegensatz zur Regierung den Gemeinwohlbeitrag 
des städtischen Wohnungsunternehmens nicht in einer Profitausrichtung zugunsten des öffentlichen Haushalts, sondern in der Bereitstellung günstigen Wohnraums für Menschen mit niedrigem Einkommen.

Ab 2014 formierte sich in Frankfurt am Main zunehmender politischer Widerstand. Die damalige Opposition im Stadtrat konnte sich allerdings mit der weitergehenden Forderung eines Mieterhöhungsverzichts nicht durchsetzen. Daraufhin bildete sich unter Beteiligung der oben genannten Bündnisse eine neue Initiative. Es kam zu einer Neusortierung der stadtpolitischen Bewegung entlang eines moderaten und eines radikaleren Flügels, wobei beide Flügel eine gewinnkritische Haltung einnahmen und eine Überwindung der neoliberalen Wohnungspolitik anstrebten (Schipper 2018).

2015 wurde das Bündnis „Eine Stadt für alle! Wem gehört die ABG?“ ins Leben gerufen, das maßgeblich für den „Mietentscheid Frankfurt“ wurde. Die Initiative beschreibt sich selbst als „Kampagne, getragen von Aktiven aus stadtpolitischen Initiativen, Stadtteilgruppen, Mieterorganisationen etc.“ (Stadt für alle o. J.). Im Jahr 2015 wurde ein erster Forderungskatalog vorgestellt, der drei Hauptforderungen enthielt: Wohnraum für Menschen mit niedrigem Einkommen zu schaffen, Ausgrenzung zu verhindern und Inklusion zu ermöglichen sowie „Mieter_innen an der Struktur und den Entscheidungen der ABG zu beteiligen, um die Gesellschaft langfristig in eine demokratische Selbstverwaltung zu überführen“ (Stadt für alle 2015). Die dritte Forderung wurde allerdings schon im folgenden Jahr abgeschwächt. Im Forderungskatalog der Frankfurter Initiativen vom November 2016 wurde zwar weiterhin auf eine soziale Wohnungspolitik und eine inklusive Stadtentwicklung gedrungen. Außerdem wurde eine Demokratisierung in Form von Beteiligung und der Etablierung von gewählten Mieter_innenbeiräten, die über Veto- und Kontrollrechte verfügen, angemahnt, um eine „Mitbestimmung und die Gestaltung des Lebensumfelds der Mieterinnen und Mieter zu gewährleisten“ (Stadt für alle 2016). Kurzfristig wurde zudem die Übertragung der Entscheidungskompetenzen des Aufsichtsrats auf das Stadtparlament gefordert. Jedoch enthält das Papier von 2016 keine konkrete Forderung zur organisationalen Umgestaltung der ABG in Richtung einer gemeinwohlorientierten Bewirtschaftung, wie sie in Form einer Stiftung oder Anstalt öffentlichen Rechts möglich gewesen wäre (ebd.).

Maßgeblich getragen von der Initiative „Eine Stadt für alle! Wem gehört die ABG?“, wurde 2018 das Bürger_innenbegehren „Mietentscheid Frankfurt“ in die Wege geleitet. Es wird von 46 Organisationen und Vereinen getragen. Daran beteiligt sind politische Parteien und Vereine des linken Spektrums, Hochschulgruppen, Gewerkschaften, aber auch Mieter_innenvereine und Kulturzentren. Für den Textvorschlag des Bürger_innenbegehrens wurden zwischen August 2018 undJanuar 2019 rund 25.000 Unterschriften gesammelt. Das Bürger_innenbegehren ist enger gefasst als die oben genannten stadtpolitischen Forderungen von 2015 und 2016 und zielt zunächst auf einen Kurswechsel der Wirtschaftspraxis der ABG ab. Es enthält drei Forderungen, eine inhaltliche Begründung sowie einen Kostendeckungsvorschlag (Mietentscheid Frankfurt o. J.). Die Forderungen lauten:

- Neubau: Zukünftig soll sich die ABG nur noch für die Errichtung von Wohnraum für Menschen aus niedrigen und mittleren Einkommensgruppen einsetzen. 
- Laufende Mietabsenkung: Die bestehenden Mieten sollen für alle Mieter_innen, die Anspruch auf eine Sozialwohnung haben, derzeit aber eine höhere Miete zahlen, auf 6,50 €/qm gesenkt werden.

- Mietabsenkung bei Neuvermietungen: Künftige Mieten im Wohnungsbestand sollen je nach Haushalt auf zwischen 6,50 und 10,50 €/qm angesetzt werden.

Derzeit ist der Status des „Mietentscheids Frankfurt“ ungeklärt. Die nötigen gültigen Stimmen wurden in der ersten Stufe des Bürger_innenbegehrens gesammelt und eine rechtliche Prüfung zur Vorbereitung des nächsten Schrittes des Bürger_innenentscheids wurde vorgenommen. Im Februar 2020 wurde allerdings vom Rat der Stadt Frankfurt beschlossen, das Bürger_innenbegehren als unzulässig abzulehnen.

In einem Rechtsgutachten der Stadt Frankfurt wurden sechs Mängel identifiziert, auf deren Basis die Weiterführung des Bürger_innenbegehrens abgelehnt wurde: Es sei zu unpräzise, die Kosten seien zu niedrig kalkuliert und die Folgekosten blieben unbenannt. Außerdem sei der Kostendeckungsvorschlag unrealistisch, Wechsel- und Folgewirkungen würden übersehen und das Bürger_innenbegehren gefährde die Aufgabenwahrnehmung von Stadt und ABG. Des Weiteren widersprächen die Vorgaben dem öffentlichen Zweck der ABG (Stadt Frankfurt 2020: 11). Die Initiative „Mietentscheid Frankfurt" hat die Entscheidung des Stadtrats angefochten und rechtliche Schritte gegen die Ablehnung des Bürger_innenbegehrens eingeleitet. Ein gerichtliches Verfahren läuft derzeit.

Im Vorfeld der Kommunalwahl 2021 signalisierten SPD und Grüne ihre Bereitschaft, einige der Forderungen der Initiative zu erfüllen. Diese beschränken sich jedoch auf die Mietpolitik der ABG, also ihre Vergabe von Wohnungen an Angehörige niedriger Einkommensgruppen. Mehr Beteiligung und Mitsprache betroffener Mieter_innen oder gar die Etablierung von Mieter_innenräten gehört - anders als noch 2015 - nicht mehr zu den Forderungen des Mietentscheids. Immerhin enthält die Frankfurter Koalitionsvereinbarung der Parlamentsmehrheit von Grünen, SPD, FDP und der Volt-Europa-Partei für die Jahre 2021 bis 2026 Quotenvorgaben für günstige Mieten in Neubauwohnungen. Mit Blick auf die ABG soll darauf hingewirkt werden, dass 60 Prozent der neu errichteten Wohnungen im geförderten Sektor gebaut werden sollen (Koalitionsvereinbarung 2021: 54).

Der Fall Frankfurt am Main verdeutlicht, dass die formal-juristische Prüfung ein wichtiges Instrument der Stadtverwaltung ist, um unerwünschte Forderungen aus der Zivilgesellschaft abzuweisen: Es wird von der Kommune als unzulässig interpretiert, die Bürger_innenschaft in einer Abstimmung zu befragen, ob die zu 100 Prozent im kommunalen Besitz befindliche Wohnungsgesellschaft von ihrer Gewinnorientierung Abstand nehmen soll. Eine direktdemokratische Abstimmung über die Neuausrichtung der Bewirtschaftung öffentlichen Eigentums wird aus formalen Gründen verhindert.

\subsection{Berlin: Rechtsetzung nach dem Mietenvolksentscheid}

Berlin ist eine Stadt mit niedriger Wohneigentumsquote und stark steigenden Mietkosten. In Westberlin wurde bis in die 1980er Jahre eine strenge 
Mietpreiskontrolle praktiziert und öffentliche Wohnungsunternehmen besaßen einen erheblichen Teil des Wohnungsbestands. In Ostberlin wurden die Wohnungsbestände durch die öffentliche Hand oder staatsnahe Organisationen kontrolliert. Zwischen Mitte der 1970er Jahre und 1990 wurden dort in großem Maßstab in Modulweise gebaute Großwohnsiedlungen errichtet (Krüger 2019), für die nach dem Ende der DDR größtenteils Kommunen oder Genossenschaften verantwortlich waren.

1990 gab es in Berlin insgesamt 20 öffentliche Wohnungsunternehmen, die 482.000 Wohnungen verwalteten (236.000 im Westen und 246.000 im Osten), was damals 28 Prozent aller Wohnungen entsprach. Bereits in den 1990er Jahren und vermehrt in den frühen 200oer Jahren wurde eine große Anzahl von Wohnungen veräußert. Bis 2005 verkaufte die Stadt mehr als 200.00o Einheiten und reorganisierte ihren Wohnungsbestand in sechs verbliebene Unternehmen. 2020 besaßen diese Unternehmen jeweils zwischen 31.00o und 75.000 Wohnungen (insgesamt 325.000 Einheiten) und sagten zu, ihren Bestand durch Bau und Kauf bis 2021 auf 360.000 Einheiten zu erweitern (Kooperationsvereinbarung 2018).

Der starke Preisanstieg der zurückliegenden Jahre, Gentrifizierung und Verdrängung erschweren es vor allem Menschen mit mittleren und geringen Einkommen sowie kleinen Gewerbetreibenden und Geflüchteten, bezahlbaren Wohnraum zu finden. Über die Jahre ist eine breite Mietenbewegung in Berlin entstanden, die als kollektiver politischer Akteur verfasst ist und sich aus institutionalisierten Interessenvertretungen sowie nachbarschaftlich und problembezogen organisierten Gruppen zusammensetzt. Es handelt sich allerdings um einen heterogenen Akteur, der durch „Fluidität und Wandelbarkeit“" gekennzeichnet ist (Vollmer 2019: 125).

Bereits 2012 hielt ein Bündnis unterschiedlicher wohnungspolitischer Initiativen eine Reihe von Konferenzen ab, um Druck auf die damalige Regierungskoalition aus SPD und CDU auszuüben. Während der Koalitionsverhandlungen wurde ein Forderungskatalog übergeben („Das mietenpolitische Dossier"), der sich insbesondere mit Konflikten um den sozialen Wohnungsbau auseinandersetzte. Da die Regierung darauf nicht einging, wurde 2015 ein Volksbegehren („Berliner Mietenvolksentscheid“) gestartet, das eine Stärkung der sozialen Wohnraumversorgung und eine Strukturreform der landeseigenen Wohnungsunternehmen anstrebte. Der Mietenvolksentscheid „sieht ebenso eine gemeinwohlorientierte Ausrichtung der landeseigenen Wohnungsunternehmen vor wie die Mitbestimmung von Mieter_innen in einem Gesamtmieterrat"(Vollmer 2019: 182). Der Mietenvolksentscheid forderte damit eine institutionalisierte Form demokratischer Mitbestimmung über Mieter_innenräte und strebte eine unternehmensrechtliche Umgestaltung der landeseigenen Wohnungsunternehmen in sechs Anstalten öffentlichen Rechts an.

Im Mittelpunkt des „Berliner Mietenvolksentscheids“ stand ein Gesetzesentwurf, der 2015 zur Abstimmung gestellt werden sollte. Er trug den Titel „Gesetz über die Neuausrichtung der sozialen Wohnraumversorgung in Berlin“ (Berliner Wohnraumversorgungsgesetz, WoVG Bln) und verfolgte einen umfassenden wohnungspolitischen Ansatz mit sechs Vorschlägen (Mietenvolksentscheid Berlin o. J.):

- Einrichtung eines öffentlichen Fonds für zukünftige Investitionen in den Wohnungsbau 
- Bereitstellung von mehr Sozialwohnungen durch Senkung der Miete für anspruchsberechtigte Haushalte

- Konzentration auf den Bau bezahlbarer Wohnungen

- Aufkauf bestehender Wohnungsbestände und Umwandlung in preisgünstigen Wohnraum

- Reorganisation der sechs städtisch kontrollierten Wohnungsunternehmen und deren Umwandlung in je eine Anstalt öffentlichen Rechts

- Demokratisierung des sozialen Wohnungsbaus durch Gewährung eines Sitzes für einen Mietervertreter im Vorstand jeder öffentlichen Wohnungsbaugesellschaft

Zwischen dem 11. April und dem 31. Mai 2015 wurden für den Mietenvolksentscheid mehr als 48.000 Unterschriften gesammelt - mehr als das Doppelte der für diese Beteiligungsstufe nötigen 20.00o Unterschriften - was die große Mobilisierung der Mieter_innenbewegung dokumentiert.

Um zu verhindern, dass die Wohnungspolitik bei den Abgeordnetenhauswahlen im Herbst 2016 zum unbeherrschbaren Thema würde, suchte die SPD nach einem Kompromiss, indem sie eine frühzeitige gesetzliche Regelung mit auf den Weg brachte, die eine Volksabstimmung überflüssig machen sollte. Zugleich wurden seitens der Verwaltung Zweifel bezüglich der Rechtsgültigkeit des vom Bündnis Mietenvolksentscheid vorgelegten Gesetzentwurfs geäußert. Diese setzten die Initiative unter Druck, dem Kompromiss zuzustimmen. In Verhandlungen zwischen der SPD und Teilen des Bündnisses wurde ein abgewandelter und abgeschwächter Gesetzesvorschlag erarbeitet, der direkt in das Parlament eingebracht und zügig verabschiedet wurde und seit 2016 gilt (WoVG Bln). Im Wesentlichen beinhaltet dieses Wohnraumversorgungsgesetz eine stark reduzierte Variante des vom Mietenvolksentscheid geforderten öffentlichen Investitionsfonds. Der Forderung nach mehr Mieter_innenmitbestimmung wurde insofern entsprochen, als dass das Gesetz die Einrichtung von Mieter_innenräten in jedem Unternehmen als Interessenvertretung der Mieter_innenschaft vorsieht und zusätzlich einer gewählten Person (ein Mitglied des Mieter_innenrats) ein Stimmrecht im Aufsichtsrat des jeweiligen Unternehmens gewährt. Allerdings sind Mieter_innenräte ehrenamtliche Organe, die ein hohes Engagement von ihren einzeln gewählten Mitgliedern erfordern und in der Praxis vor allem konsultativen Charakter haben.

Die Rechtsform der sechs öffentlichen Wohnungsunternehmen blieb dagegen unangetastet, diese sind weiterhin als Aktiengesellschaften bzw. Gesellschaften mit beschränkter Haftung verfasst und damit an gesellschaftsrechtliche Vorgaben gebunden, die eine gemeinwirtschaftliche Praxis erschweren. Allerdings wurde mit der Wohnraumversorgung Berlin (WVB) eine Anstalt öffentlichen Rechts gegründet, deren Aufgabe eine stärkere politische Steuerung der sechs öffentlichen Landeswohnungsunternehmen ist (Kuhnert 2016). Laut Gesetz ist die WVB dafür zuständig, politische Leitlinien in Bezug auf den Versorgungs- und Wohnungsmarktauftrag der landeseigenen Unternehmen zu entwickeln. Sie verfügt allerdings nicht über Vermögen und hat vor allem beratenden Charakter.

Das Zustandekommen des Wohnraumversorgungsgesetzes, insbesondere die von der Politik vorangetriebenen Verhandlungen mit Teilen der 
zivilgesellschaftlichen Bewegung, wurde zwar von verschiedenen Akteur_ innen begrüßt, führte aber auch zu einer Zersplitterung des Netzwerks (Junker/Raab/Schurian 2016). Während die eine Seite argumentierte, dass zuvor übersehene rechtliche Mängel das gesamte Unterfangen hätten gefährden können, kritisierten andere den Kompromiss als nicht weitgehend genug und als vorzeitigen Ausverkauf der Bewegung (Diesselhorst 2018).

Die anschließende Abgeordnetenhauswahl im September 2016 brachte eine in Fragen der Wohnungspolitik reformorientierte Mehrheit aus SPD, LINKEN und Bündnis 90/Die Grünen hervor. Bei der sozialen Bewirtschaftung und preisgünstigem Neubau setzte die neue Regierungskoalition verstärkt auf die sechs landeseigenen Wohnungsunternehmen, auch wenn grundlegende Eingriffe in die Unternehmenspraxis nicht stattfanden. Die Steuerung der Unternehmen erfolgte über eine politische Vereinbarung, die sogenannte Kooperationsvereinbarung, auf die sich die Unternehmen mit dem Berliner Senat geeinigt hatten. Sie enthält politische Zielvorgaben zur sozialen Bestandsbewirtschaftung, schreibt in Teilen die Miethöhe fest, regelt Partizipationsfragen und definiert Ziele der Ausweitung des Wohnungsbestands (Kooperationsvereinbarung 2018). Gemeinsam mit der zuständigen Senatorin Katrin Lompscher (DIE LINKE), die über enge Beziehungen zur stadtpolitischen Bewegung verfügte, wurde ein Kurswechsel in der Wohnungspolitik eingeleitet, der über eine sozialere Ausrichtung der Bewirtschaftungspraxis der landeseigenen Unternehmen hinausging. Im Februar 2020 trat das Gesetz zur Mietenbegrenzung im Wohnungswesen in Berlin (Mietendeckel) in Kraft, das ein gesetzliches Einfrieren der Mieten für die meisten Wohnungen in der Stadt erwirkte, in einigen Fällen sogar die Absenkung von Bestandsmieten. Dies galt auch für die landeseigenen Unternehmen. Im Frühjahr 2021 wurde das Gesetzjedoch durch eine Entscheidung des Bundesverfassungsgerichts aus Gründen der mangelnden Zuständigkeit des Landes Berlin gekippt. Einzelaspekte sollen aber für die landeseigenen Unternehmen weiterhin gelten.

Der neueste und vielleicht spektakulärste Erfolg der Berliner Mieter_innenbewegung ist das Volksbegehren „Deutsche Wohnen \& Co. enteignen“. In einer ersten Stufe wurden in rund acht Wochen mehr als 70.00o Unterschriften gesammelt. Nach einer langen juristischen Prüfphase durch den Senat lief im Frühjahr und Sommer 2021 die zweite Phase der Unterschriftensammlung. Die notwendige Unterschriftenzahl (7 \% der Wahlberechtigten) wurde weit übertroffen, sodass es im September 2021 zu einem Volksentscheid über einen „Beschluss zur Erarbeitung eines Gesetzentwurfs durch den Senat zur Vergesellschaftung der Wohnungsbestände großer Wohnungsunternehmen (Vergesellschaftungsgesetz)“(Landeswahlleiter Berlin 2021) kam - so der Titel des zur Abstimmung vorgelegten Beschlusses, der mit über 57 Prozent der Stimmen angenommen wurde. Der Senat von Berlin ist nun aufgefordert, einen Gesetzentwurf zur Vergesellschaftung der Wohnungsbestände großer Wohnungsunternehmen zu erarbeiten.

Der Fall Berlin verdeutlicht den hohen Aufwand langjähriger Vernetzung und Kooperation der mietenpolitischen Akteur_innen, zeigt aber auch, dass es möglich ist, die politische Problemdeutung zu verschieben und Druck auf etablierte politische Kräfte auszuüben. Auch wenn Forderungen des Mietenvolksentscheids abgeschwächt wurden, sind in Berlin durch 
verschiedene Maßnahmen soziale Belange der Wohnraumbewirtschaftung und Möglichkeiten der Mieter_innenmitbestimmung institutionalisiert worden: per Gesetz, als Vereinbarung zwischen den Unternehmen und dem Senat sowie in Form der Wohnraumversorgung Berlin wurden konkrete Regelungen festgeschrieben. Dies kann als Erfolg der mietenpolitischen Bewegung gedeutet werden. Ob und in welcher Form er ausgeweitet werden muss, ist an anderer Stelle zu untersuchen.

\section{Auswertung: Vergleich Frankfurt am Main und Berlin}

Der Fallvergleich zeigt einige Parallelen zwischen Frankfurt am Main und Berlin auf: Zunächst lässt sich festhalten, dass gut organisierte Initiativen und stadtweite Netzwerke in der Lage sind, direktdemokratische Verfahren zur Neuausrichtung der öffentlichen Wohnungsunternehmen anzustoßen und durchzuführen. In beiden Fällen wurden die nötigen Unterschriften zur Einleitung der jeweils nächsten Stufe des Bürger_innen-bzw. Volksbegehrens erreicht. Dies zeigt das Mobilisierungspotenzial für wohnungspolitische Themen. Beide Initiativen waren erfolgreich darin, die Wirtschaftspraxis kommunaler Wohnungsunternehmen zum Gegenstand der öffentlichen Debatte zu machen. Allerdings stießen sie dabei auf den Widerstand der jeweiligen Stadtverwaltungen, die Zweifel hinsichtlich der Rechtmäßigkeit der Begehren äußerten. Die formale juristische Prüfung stellt eine ernst zu nehmende Hürde für direktdemokratische Bestrebungen dar, insbesondere wenn die Verantwortlichen in den Verwaltungen eine restriktive Auslegung praktizieren. Dies unterstreicht die Bedeutung von juristischem Fachwissen auch für zivilgesellschaftliche Bewegungen. Der Frankfurter Fall zeigt, dass auch rechtliche Klagen seitens der Mietenbewegung zum Repertoire der wohnungspolitischen Auseinandersetzungen zählen. Dort hat das Bündnis Mietentscheid Widerspruch gegen den negativen Ratsbeschluss eingelegt und erhofft sich eine Korrektur der Ablehnungsentscheidung.

Die Entwicklungen unterscheiden sich aber auch in einigen Aspekten, allen voran in der Reichweite der Forderungen und deren Durchsetzung. Diese Differenzen lassen sich anhand von drei Dimensionen systematisieren: Art der Bewirtschaftung, Mieter_innenmitbestimmung und Steuerungsregime der öffentlichen Unternehmen bzw. deren Rechtsform. In beiden Städten zielten die Bürger_innenbegehren auf eine Umsteuerung der Wohnraumbewirtschaftung, insbesondere auf eine stärkere Ausrichtung der Wohnraumvergabe an den Bedürfnissen von Menschen mit geringen und mittleren Einkommen. Die Berliner Initiative ging allerdings deutlich über Forderungen einer veränderten Bewirtschaftung hinaus, sie strebte zusätzliche Finanzmittel, eine institutionalisierte Beteiligung der Mieter_innenschaft in unternehmensweiten Mieter_innenräten sowie eine Veränderung der Rechtsform der Unternehmen an. Auch wenn diese Forderungen im Verlauf des Gesetzgebungsverfahrens abgeschwächt wurden - insbesondere die finanzielle Zusatzförderung und veränderte Rechtsformen haben sich nicht durchgesetzt -, konnten in Berlin doch einige Schritte im Sinne der Initiativen vollzogen werden. Dies betrifft vor allem die Mitbestimmung in Form von Mieter_innenräten, die in Frankfurt am Main zwar zu Beginn Teil der Forderungen der Mietenbewegung war, später aber keinen Eingang in das Bürger_innenbegehren gefunden hat.[3] 
In Berlin ist die institutionalisierte Vertretung von Repräsentant_innen der Mieter_innenschaft in den Aufsichtsräten der jeweiligen Wohnungsgesellschaften mittlerweile gesetzlich abgesichert. Zum einen wurden bereits existierende dezentrale Mieter_innenbeiräte gestärkt, zum anderen wurden firmenweite Mieter_innenräte eingeführt, die laut Senatsverwaltung die Mitbestimmung stärken und als Instrument einer „Demokratisierung des Wohnens" dienen (Senatsverwaltung für Stadtentwicklung und Wohnen o. J. a). Allerdings handelt es sich vor allem um eine konsultative Form der Beteiligung, bei der Anregungen aus der Mieter_innenschaft gebündelt und Vorschläge unterbreitet werden, um auf diese Weise zum Interessenausgleich beizutragen. Das Wohnraumversorgungsgesetz sieht allerdings auch vor, dass Mieter_innenräte sich mit Unternehmensplanungen bei den Neubau-, Modernisierungs- und Instandsetzungsprogrammen und der Quartiersentwicklung befassen und dazu Stellung nehmen können.

Außerdem wurden im Berliner Fall die unternehmensrechtlichen Grundsätze der öffentlichen Gesellschaften infrage gestellt. Zwar wurden die sechs landeseigenen Gesellschaften nicht wie gefordert in Anstalten des öffentlichen Rechts (AöR) umgewandelt, es wurde aber eine neue AöR gegründet, die „Wohnraumversorgung Berlin“. Ihre Aufgabe ist es, unternehmenspolitische Leitlinien bezüglich der Wahrnehmung des Versorgungs- und Wohnungsmarktauftrags der landeseigenen Wohnungsunternehmen für die Landesregierung zu entwickeln, zu prüfen und fortzuschreiben (Senatsverwaltung für Stadtentwicklung und Wohnen o. J. b). Die Stadt verfügt somit über eine wohnungspolitische Koordinationsstelle, um eine gemeinwohlorientierte Ausrichtung der landeseigenen Gesellschaften zu stärken. Tabelle 2 enthält eine zusammenfassende Übersicht der zentralen Forderungen.

In Berlin ist es möglich, über landesrechtliche Kompetenzen Veränderungen bei den Wohnungsunternehmen gesetzlich zu verankern. Allerdings sind dabei immer gleichzeitig sechs zu steuernde Unternehmen betroffen. In Frankfurt am Main ist dagegen nur ein städtisches Unternehmen Objekt des Bürger_innenbegehrens, sodass ein organisationaler Wandel bereits über Veränderungen im Gesellschaftervertrag zwischen Stadt und ABG herbeigeführt werden könnte. Dagegen sprechen allerdings die politischen Mehrheiten, die von einem stärkeren Gewicht bürgerlicher Parteien geprägt

\begin{tabular}{|c|c|c|}
\hline & Mietentscheid Frankfurt/Main & Mietenvolksentscheid Berlin \\
\hline angestrebte Bewirtschaftung & $\begin{array}{l}\text { - Wohnungsvergabe auf Basis } \\
\text { sozialer Kriterien } \\
\text { moderat profitorientiert: } \\
\text { Überschussabführung an } \\
\text { Eigentümerin (Stadt) bleibt } \\
\text { bestehen }\end{array}$ & $\begin{array}{l}\text { - Wohnungsvergabe auf Basis } \\
\text { - } \quad \text { bezialer Kriterien } \\
\text { von Eigenkapital durch } \\
\text { Eigentümerin (Stadt), } \\
\text { Ausweitung der Förderung }\end{array}$ \\
\hline Mieter_innenmitbestimmung & - $\quad$ nicht vorgesehen & $\begin{array}{l}\text { - institutionalisiert: lokale } \\
\text { Mieter_innenbeiräte und } \\
\text { Mieter_innenräte auf } \\
\text { Unternehmensebene }\end{array}$ \\
\hline $\begin{array}{l}\text { Steuerungsregime der } \\
\text { öffentlichen Unternehmen / } \\
\text { Rechtsform }\end{array}$ & $\begin{array}{l}\text { - bleibt unangetastet } \\
\text { (traditionelle kommunale } \\
\text { Eigentumssteuerung durch } \\
\text { städtische Entscheidungs- } \\
\text { träger_innen) }\end{array}$ & $\begin{array}{l}\text { - Umstrukturierung (neue } \\
\text { (Selbst-)Steuerung in Form } \\
\text { von Anstalten öffentlichen } \\
\text { Rechts }\end{array}$ \\
\hline
\end{tabular}

Tab. 2 Übersicht der direktdemokratischen Forderungen (Quelle: eigene Darstellung) 
sind, auch wenn der Koalitionsvertrag von 2021 für die ABG eine sozialere Ausrichtung der Wohnraumbewirtschaftung vorsieht.

Abschließend lässt sich festhalten, dass das Frankfurter Bürger_innenbegehren größeren Herausforderungen gegenüberstand und einen enger gefassten Forderungskatalog zur Abstimmung gestellt hat. In Berlin war das politische Klima günstiger, der Anspruch der Neuausrichtung der Unternehmen aber auch größer. Auch wenn die Berliner Initiative während der Verhandlungen zum Gesetzentwurf Abstriche machen musste, insbesondere bei der Umwandlung der Rechtsform, zeigt sich doch, dass das Thema des grundlegenden Wandels öffentlicher Unternehmen erfolgreich auf die politische Agenda gesetzt werden kann. Wie nachhaltig die konkreten Veränderungen sind, ob sie beibehalten oder erweitert werden, ist derzeit noch nicht abzusehen. Die Initiative „Deutsche Wohnen \& Co. enteignen“ jedenfalls hat die Frage nach der Rechts- und Eigentumsform großer Wohnraumbestände wieder aufgegriffen und spitzt sie weiter zu.

\section{Fazit und Ausblick: Neuer Umgang mit öffentlichem Eigentum}

In Frankfurt am Main und Berlin zeigt sich, dass die Ausrichtung der öffentlichen Wohnungsunternehmen umstritten ist. Konflikte und Auseinandersetzungen zeigen sich insbesondere mit Blick darauf, welche Anspruchsgruppen Berücksichtigung finden sollen und wessen Wohl bevorzugt in den Blick genommen wird. In Frankfurt am Main verteidigte die Ratsmehrheit den Status quo mit dem Verweis darauf, dass Gewinne der ABG an die Stadt flössen und somit allen zugutekämen. Hier wird ein Gemeinwohlverständnis formuliert, das das Mehrheitsinteresse mit den Erfordernissen der städtischen Haushaltspolitik in Einklang bringt. Dies ist ein Beispiel dafür, dass der Verweis auf das Gemeinwohl auch verwendet werden kann, um eine profitorientierte Wohnungspolitik zu legitimieren,[4] während Forderungen nach bezahlbarer/sozialer Wohnraumversorgung als Vertretung von Partikularinteressen deklariert werden. Ein solches Verständnis bleibt den Begründungszusammenhängen neoliberaler Stadtpolitiken verhaftet.

Aber auch in Berlin bleibt die unternehmensrechtliche Ausrichtung der Wohnungsunternehmen umstritten. Insbesondere durch die Beibehaltung der Rechtsform öffentlicher Wohnungsunternehmen als Aktiengesellschaften bzw. Gesellschaften mit beschränkter Haftung wird eine (zukünftige) profitorientierte Bewirtschaftungspraxis nicht ausgeschlossen. Gemeinwohl bleibt also auch hier ein umkämpfter Gegenstand, der fortwährend situativ und zeitlich begrenzt zwischen Akteur_innen in ungleichen Machtverhältnissen ausgehandelt wird - und werden muss.

Direktdemokratische Verfahren sind dabei eine Möglichkeit, einen grundlegenden Wandel öffentlicher Unternehmen herbeizuführen, indem sie ein Gemeinwohlverständnis auf die Agenda setzen, das folgende Aspekte umfasst: die Art der Bewirtschaftung, die Mitbestimmung von Mieter_innen und die rechtliche Form der Unternehmenssteuerung. In den bisherigen Diskussionen und Auseinandersetzungen hat die soziale Wohnraumversorgung - oft vor dem Hintergrund unmittelbarer sozialer Härten - den größten Raum eingenommen. Ein grundlegender Paradigmenwechsel in der Wohnungspolitik erscheint aber 
ohne eine Institutionalisierung der Mieter_innenmitbestimmung (von Vollmer und Kadi (2018) als „Governance“ kategorisiert) und ohne eine langfristige gesellschaftsrechtliche Umstrukturierung der Firmen auf nicht-profitorientierte Formen des Wirtschaftens wenig aussichtsreich (vgl. Jensen 2021).

Eine dauerhafte Mieter_innenmitbestimmung könnte - analog zur gesetzlichen Arbeitnehmer_innenmitbestimmung über Betriebsräte - als Ko-Management der Mieter_innenschaft an bisher schon erfolgreiche Formen bundesrepublikanischer Wirtschaftspraxis anknüpfen. Bisher sind die Erfahrungen zwar gering, aber die zunehmend eng vernetzten stadtpolitischen Initiativen könnten eine wichtige Rolle bei der Unterstützung der einzelnen Mieter_innenräte spielen. Eine umfassende und institutionalisierte Mieter_innenmitbestimmung, wie sie etwa vom Bündnis „kommunal \& selbstverwaltet Wohnen“ in Berlin gefordert wird, umfasst alle Bereiche des Wohnens (Planung, Verwaltung, Modernisierung, Nachbarschaftsbeziehungen und mögliche Veräußerung) und betont neben der Mitbestimmung kommunaler Unternehmen auch die Bedeutung der Selbstverwaltungspraxis, wie sie in vielen Hausprojekten stattfindet (kusWo 2018).

Für eine langfristig sozial orientierte Wohnraumversorgungspraxis durch öffentliche Unternehmen spielt deren Rechtsform eine wichtige Rolle, denn auch wenn Unternehmen sich vollständig in öffentlichem Besitz befinden, unterliegen sie zugleich dem Gesellschaftsrecht (Aktien- oder GmbHGesetz). Das erschwert demokratische Teilhabe und Transparenz, etwa wenn Geschäftsgeheimnisse berührt sind oder Vorstände mit umfangreichen Kompetenzen ausgestatten werden. Die langfristige Neuausrichtung öffentlicher Unternehmen dürfte die größte Herausforderung darstellen: Auf der einen Seite erfordert sie einen organisationalen Kulturwandel, auf der anderen Seite bleibt die Bewirtschaftung von Wohnraum eine anspruchsvolle Tätigkeit, die Sachkenntnis und professionelles Handeln erfordert. Eine erfolgreiche Umwandlung von Unternehmen in eine andere Rechtsform, wie etwa eine Anstalt öffentlichen Rechts oder eine Stiftung, kann zudem nur durch die Eigentümer_innen erfolgen. Solange eine Rechtsformänderung nicht durchsetzbar ist, könnte die Wiedereinführung der Wohnungsgemeinnützigkeit mithilfe des Steuerrechts eine wichtige Unterstützung bieten (vgl. Holm/Horlitz/Jensen 2017; Kuhnert/Leps 2017).

Bürger_innen-bzw. Volksbegehren sind also ein wichtiges Instrument für zivilgesellschaftliche Initiativen, denn mit der Formulierung konkreter Vorschläge, Forderungen und Gesetze können sie unmittelbaren politischen Druck in ausgewählten Sachfragen aufbauen. Auch juristische Klagen und andere rechtliche Mittel gehören zum strategischen Repertoire stadtpolitischer Bewegungen (Kusiak 2021). Direktdemokratische Verfahren bieten sich aber nicht nur als Form konkreter Kritik an der lokalen Wohnungspolitik an, sondern können für die Stadtgesellschaft auch ein Weg der Selbstermächtigung sein, die den wohnungspolitischen Diskurs verschiebt und soziale Bedürfnisse offensiv gegen eine profitorientierte Organisationspraxis öffentlicher Unternehmen stellt. Die Vielzahl von Initiativen aus unterschiedlichen Großstädten zeigt, dass es sich nicht um isolierte Ereignisse handelt. Diese Vielfalt ermöglicht es den Initiativen auch, voneinander zu lernen und durch gezielte Vernetzung den Transfer von Ideen, Strategien und Konzepten herbeizuführen (Rink/Vollmer 2019). 
Allerdings sind direktdemokratische Initiativen, dieeinen anderen Umgang mit öffentlichem Eigentum herbeiführen wollen, voraussetzungsvoll. Die Hürden für die Durchführung von Bürger_innen- und Volksbegehren sind hoch und erfordern umfangreiche juristische, politische und ökonomische Sachkenntnis. Außerdem ist der logistische Aufwand erheblich - in wenigen Monaten ausreichend gültige Unterschriften müssen gesammelt werden. All dies ist vornehmlich ehrenamtlich organisiert und erfordert ein großes Engagement der Beteiligten. Zugleich treffen die Begehren oftmals auf Widerstände in der Stadtverwaltung und auch bei den öffentlichen Unternehmen selbst. In Frankfurt am Main ist dies umfänglich dokumentiert, aber auch in Berlin hat sich - trotz weitergehender gesetzlicher Vorgaben - am Selbstverständnis der öffentlichen Unternehmen und ihrer Geschäftsführer_ innen bisher wenig geändert.[5]

Die angespannten Wohnungsmärkte in deutschen Städten zeigen, dass es bisher nicht gelingt, eine grundlegende Wende in der Wohnungspolitik herbeizuführen. Die Frage, welchen Beitrag öffentliche Unternehmen zur Entspannung der Wohnungsmarktlage leisten können, wird damit besonders dringlich. Die Fallanalysen haben klargemacht, dass in der neoliberalen Stadt nicht davon auszugehen ist, dass öffentliche Eigentümer_innenschaft eine umfassende Gemeinwohlorientierung der Wohnraumbewirtschaftung sicherstellt. Die Initiativen haben sich dafür eingesetzt, dass soziale Wohnraumbewirtschaftung, Mieter_innenbeteiligung und alternative Rechtsformen entscheidende Bestandteile einer solchen Gemeinwohlorientierung sind. In welcher Weise sie zur Geltung kommen, hängt davon ab, wie gut sie sich mit ihren stadtpolitischen Forderungen durchsetzen können. Dabei spielt die Institutionalisierung von erreichten Erfolgen eine wichtige Rolle. Gemeinwohl in der Wohnungspolitik wird weiter umkämpft bleiben, aber die Chancen für die Initiativen, ihre Forderungen durchzusetzen, steigen, wenn sie gut vernetzt und organisiert sind. Der Umgang mit öffentlichem Eigentum ist dabei eine zentrale Facette zur Überwindung der neoliberalen Stadt.

\section{Endnoten}

[1] Bürger_innen- und Volksbegehren sind demokratische Initiativen, die sich auf die Regelungen eines konkreten Sachverhalts richten. Die kommunal- und landesrechtlichen Vorgaben variieren je nach den gesetzlichen und verfassungsrechtlichen Bestimmungen. In der Regel richten sich Bürger_innenbegehren an diekommunale Ebene, Volksbegehren an die Landesebene.

[2] Diese formalen Unterschiede der Fälle schlagen sich zwar in den rechtlichen Vorgaben für die Beteiligungsverfahren nieder, spielen aber unserer Einschätzung nach für die Erklärung der Unterschiede zwischen beiden Fällen keine entscheidende Rolle.

[3] Mieter_innenräte, die einen Sitz im Aufsichtsrat einer öffentlichen Gesellschaft wahrnehmen und damit an Unternehmensentscheidungen beteiligt sind, sind bisher sehr selten. Ein prominentes Beispiel findet sich in der unmittelbaren Nähe von Frankfurt am Main: Bei der Wohnbau Gießen GmbH sind seit den 1990er Jahren Vertreter_innen der Mieter_innenschaft im Aufsichtsrat (Kuhnert/Leps 2017: 320 f.). Die Verankerung solcher Entscheidungsbefugnisse ist durch eine Änderung des Gesellschaftervertrags möglich und wäre auch eine Option für mehr Mitbestimmung bei der ABG.

[4] Dass Überschüsse auch stadtweit oder sogar bundesweit zweckgebunden für eine Expansion der sozialen Wohnraumversorgung verwendet werden könnten, zeigt der 
Vorschlag eines revolvierenden Fonds, der ein Grundpfeiler des Konzepts der Neuen Gemeinnützigkeit ist (siehe Holm/Horlitz/Jensen 2017: 27 f.).

[5] Die Bedingungen für einen organisationalen Kultur- und Führungsstilwandel innerhalb der öffentlichen Wohngesellschaften sind allerdings weitgehend unerforscht. Insbesondere Forschung zur Rolle des leitenden Managements bei der Umstrukturierung und Neuausrichtung öffentlicher Unternehmen ist ein Desiderat.

\section{Autor innen}

Sebastian Botzem ist Politikwissenschaftler und Ökonom. Er arbeitet zu den Themen Internationalisierung des Finanzwesens, wirtschaftliche Globalisierung, ökonomisches Denken und Finanzialisierung der Wohnungswirtschaft.

sebastian.botzem@uni-bremen.de

Natalia Besedovsky ist Soziologin. Sie arbeitet zu den Themen Wirtschafts- und Wissenssoziologie, Praktiken in der Finanzwirtschaft sowie Finanzialisierung der Nachhaltigkeit. natalia.besedovsky@uni-hamburg.de

\section{Literatur}

Aalbers, Manuel B. (2017): The variegated financialization of housing. In: International Journal of Urban and Regional Research 41/4, 542-554.

ABG (2016): Wohnen für Alle. 125 Jahre ABG FRANKFURT HOLDING. https://www.abg. de/unternehmen/wir-ueber-uns/chronik.php (letzter Zugriff am 28.8.2020).

BBSR - Bundesinstitut für Bau-, Stadt- und Raumforschung (2019): Gemeinwohlorientierte Wohnungspolitik - Stiftungen und weitere gemeinwohlorientierte Akteure. Handlungsfelder, Potenziale und gute Beispiele. Bonn: Bundesamt für Bauwesen und Raumordnung.

Botzem, Sebastian / Dobusch, Leonhard (2012): Dienstleister der Finanzialisierung. Fragmentierte Organisation und kalkulierte Profite in der Immobilienwirtschaft. In: Kölner Zeitschrift für Soziologie und Sozialpsychologie 64/4, 673-700.

Deutscher Bundestag (2020): Zielerreichung der Bundesregierung, für bezahlbare Mieten zu sorgen. Antwort der Bundesregierung auf die Kleine Anfrage der Abgeordneten Christian Kühn (Tübingen), Daniela Wagner, Canan Bayram, weiterer Abgeordneter und der Fraktion BÜNDNIS 90/DIE GRÜNEN - Drucksache 19/18230. https://dip21. bundestag.de/dip21/btd/19/182/1918230.pdf (letzter Zugriff am 23.9.2020).

Diesselhorst, Jonathan (2018): Wenn stadtpolitische Bewegungen das Terrain des Staats betreten. In: PROKLA. Zeitschrift für kritische Sozialwissenschaft 48/191, 265-282.

Döring, Nicola / Bortz, Jürgen (2016): Forschungsmethoden und Evaluation in den Sozialund Humanwissenschaften. Berlin: Springer.

Gennies, Mona (2021): Konzeptverfahren als Instrument einer gemeinwohlorientierten Stadtentwicklung. Technische Universität Berlin: ISR Impulse Online Nr. 68. https:// verlag.tu-berlin.de/produkt/978-3-7983-3174-7/ (letzter Zugriff 30.4.2021).

Gestring, Norbert / Ruhne, Renate / Wehrheim, Jan (Hg.) (2014): Stadt und soziale Bewegungen. Wiesbaden: Springer Fachmedien.

Heeg, Susanne (2013): Wohnungen als Finanzanlage. Auswirkungen von Responsibilisierung und Finanzialisierung im Bereich des Wohnens. In: sub \urban. zeitschrift für kritische stadtforschung 1/1, 75-99.

Held, Tobias (2011): Verkäufe kommunaler Wohnungsbestände. Ausmaß und aktuelle Entwicklungen. In: Informationen zur Raumentwicklung 12/2011, 675-682.

Hilbrandt, Hanna (2019): Everyday urbanism and the everyday state. Negotiating habitat in allotment gardens in Berlin. In: Urban Studies 56/2, 352-367.

Holm, Andrej (2011): Wohnung als Ware. Zur Ökonomie und Politik der Wohnungsversorgung. In: Widersprüche: Zeitschrift für sozialistische Politik im Bildungs-, Gesundheitsund Sozialbereich 31/121, 9-20.

Holm, Andrej (2013): Wohnen als soziale Infrastruktur. In: Z. Zeitschrift marxistische Erneuerung 95. http://www.zeitschrift-marxistische-erneuerung.de/article/1102. wohnen-als-soziale-infrastruktur.html/ (letzter Zugriff am 10.5.2021). 
Holm, Andrej (2014): Das Recht auf die Stadt in umkämpften Räumen. Zur gesellschaftlichen Reichweite lokaler Proteste. In: Norbert Gestring / Renate Ruhne / Jan Wehrheim (Hg.), Stadt und soziale Bewegungen. Wiesbaden: Springer Fachmedien, 43-62.

Holm, Andrej / Horlitz, Sabine / Jensen, Inga (2015): Neue Gemeinnützigkeit. Gemeinwohlorientierung in der Wohnungsversorgung. Arbeitsstudie im Auftrag der Fraktion DIE LINKE. im deutschen Bundestag, Berlin.

Holm, Andrej / Horlitz, Sabine / Jensen, Inga (2017): Neue Wohnungsgemeinnützigkeit. Voraussetzungen, Modelle und erwartete Effekte. Studie im Auftrag der Rosa-LuxemburgStiftung, Berlin.

Horlitz, Sabine (2019): Community Land Trusts. Nachbarschaftliche Selbstverwaltung gegen Bodenspekulation und Verdrängung. In: Brigitta Gerber / Ulrich Kriese (Hg.), Boden behalten, Stadt gestalten, Zürich: Rüffer und Rub, 105-115.

Hunger, Bernd (2017): Für und Wider Wohnungsgemeinnützigkeit. In: Barbara Schönig / Justin Kadi / Sebastian Schipper (Hg.), Wohnraum für alle?! Perspektiven auf Planung, Politik und Architektur, Bielefeld: transcript, 153-164.

Jensen, Inga (2021): Wohnraum als soziale Infrastruktur. In: Barbara Schönig / Lisa Vollmer (Hg.), Wohnungsfragen ohne Ende?!, Bielefeld: transcript, 147-162.

Junker, Stephan / Raab, Susanna / Schurian, Hannah (2016): Wundermittel Volksentscheid? Chancen und Grenzen für die mietenpolitische Bewegung. In: LuXemburg. Gesellschaftsanalyse und linke Praxis September 2016, 114-121.

Kamleithner, Christa (2009): „Regieren durch Community“. Neoliberale Formen der Stadtplanung. In: Matthias Drilling / Olaf Schnur (Hg.), Governance der Quartiersentwicklung. Theoretische und praktische Zugänge zu neuen Steuerungsformen. Wiesbaden: VS Verlag für Sozialwissenschaften, 29-47.

Koalitionsvereinbarung (2021): Ein neues Frankfurt gestalten. Koalitionsvertrag für Frankfurt 2021-2026. Bündnis 90/Die Grünen, SPD, FDP, Volt Frankfurt. https:// dynamic.faz.net/download/2021/Koalitionsvertrag_2021_2026.pdf (letzter Zugriff am 23.5.2021).

Kooperationsvereinbarung (2018): Bericht zur Kooperationsvereinbarung 2018. „Leistbare Mieten, Wohnungsneubau und soziale Wohnraumversorgung“ über das Jahr 2018. Berlin: Wohnraumversorgung Berlin - Anstalt öffentlichen Rechts.

Krüger, Arvid (2019): Die veränderte Rolle der Wohnungswirtschaft in den Großen Siedlungen. In: Uwe Altrock / Detlef Kurth / Ronald Kunze (Hg.), Programmatik der Stadterneuerung. Wiesbaden: Springer Fachmedien, 25-47.

Kuhnert, Jan (2016): Soziale Aufgaben für öffentliche Wohnungsunternehmen. Gesetz erzwingt Neuorientierung der Wohnungsunternehmen von Berlin. In: Forum Wohnen und Stadtentwicklung 17/2, 63-68.

Kuhnert, Jan / Leps, Olof (2017): Neue Wohnungsgemeinnützigkeit. Wege zu langfristig preiswertem und zukunftsgerechtem Wohnraum. Wiesbaden: Springer.

Kusiak, Joanna (2021): Trespassing on the law. Critical legal engineering as a strategy for action research. In: Area. https://doi.org/10.1111/area.12700, 1-8.

kusWo - kommunal \& selbstverwaltet Wohnen (2018): Broschüre. https://kommunalselbstverwaltet-wohnen.de/2018_kuswo_broschuere.pdf (letzter Zugriff am 15.5.2021).

Künkel, Jenny (2018): Die städtische Produktion von „Armutsmigration“. In: PROKLA. Zeitschrift für kritische Sozialwissenschaft 48/191, 283-298.

Landeswahlleiter Berlin (2021): Volksentscheid über einen Beschluss zur Erarbeitung eines Gesetzentwurfs durch den Senat zur Vergesellschaftung der Wohnungsbestände großer Wohnungsunternehmen. https://www.berlin.de/wahlen/abstimmungen/deutschewohnen-und-co-enteignen/artikel.1040424.php (Letzter Zugriff am 30.09.2021)

Lebuhn, Henrik (2008): Stadt in Bewegung. Mikrokonflikte um den öffentlichen Raum in Berlin und Los Angeles. Münster: Westfälisches Dampfboot.

Mayntz, Renate (1992): Interessenverbände und Gemeinwohl - Die Verbändestudie der Bertelsmann Stiftung. In: Renate Mayntz (Hg.), Verbände zwischen Mitgliederinteressen und Gemeinwohl. Gütersloh: Bertelsmann Stiftung, 11-35.

Mayntz, Renate (2001): Wohlfahrtsökonomische und systemtheoretische Ansätze zur Bestimmung von Gemeinwohl. In: Herfried Münkler / Karsten Fischer (Hg.), Gemeinwohl und Gemeinsinn. Rhetoriken und Perspektiven sozial-moralischer Orientierung. Berlin: Akademie Verlag, 111-126.

Mayring, Philipp (2002): Einführung in die qualitative Sozialforschung. Eine Anleitung zu qualitativem Denken. Weinheim/Basel. 
Mehr Demokratie (2020): Bürgerbegehrensbericht 2020. https://www.mehr-demokratie. de/themen/buergerbegehren-in-den-kommunen/buergerbegehrensbericht-2020/ (letzter Zugriff am 29.09.2021).

Mehr Demokratie (2021): Datenbank Bürgerbegehren. https://www.mehr-demokratie. $\mathrm{de} / \mathrm{nc} /$ datenbank-buergerbegehren/?tx_buergerbegehren_list\%5Baction $\% 5 \mathrm{D}=$ list\&tx_ buergerbegehren_list\%5Bcontroller\%5D=Initiative\&cHash=8add6fde8bbd4b5b1b272cb bobod2dd4/ (letzter Zugriff am 11.5.2021).

Mertens, Daniel (2015): Erst sparen, dann kaufen? Privatverschuldung in Deutschland. Frankfurt am Main: Campus.

Metzger, Joscha / Schipper, Sebastian (2017): Postneoliberale Strategien für bezahlbaren Wohnraum? Aktuelle wohnungspolitische Ansätze in Frankfurt am Main und Hamburg. In: Barbara Schönig / Justin Kadi / Sebastian Schipper (Hg.), Wohnraum für alle?! Perspektiven auf Planung, Politik und Architektur. Bielefeld: transcript, 178-212.

Metzger, Philipp (2020): Die Finanzialisierung der deutschen Ökonomie am Beispiel des Wohnungsmarktes. Münster: Westfälisches Dampfboot.

Mietentscheid Frankfurt (o. J.): Sind Sie dafür, dass die Stadt Frankfurt am Main... https:// mietentscheid-frankfurt.de/?page_id=323 (letzter Zugriff am 17.9.2020).

Mietenvolksentscheid Berlin (o. J.): Gesetz über die Neuausrichtung der sozialen Wohnraumversorgung in Berlin (Berliner Wohnraumversorgungsgesetz). https:// mietenvolksentscheidberlin.de/wp-content/uploads/2015/03/150308c-WoVG-Bln-mitBegruendung.pdf (letzter Zugriff am 17.9.2020).

Mittendorf, Volker / Schiller, Theo (2008): Initiative und Referendum. In: Norbert Kersting $(\mathrm{Hg})$, Politische Beteiligung. Einführung in dialogorientierte Instrumente politischer und gesellschaftlicher Partizipation. Wiesbaden: Springer, 142-156.

Münkler, Herfried / Bluhm, Harald (Hg.) (2001): Gemeinwohl und Gemeinsinn. Historische Semantiken politischer Leitbegriffe. Berlin: Akademie Verlag.

Münkler, Herfried / Fischer, Karsten (1999): Gemeinwohl und Gemeinsinn: Thematisierung und Verbrauch soziomoralischer Ressourcen in der modernen Gesellschaft. Berichte und Abhandlungen / Berlin-Brandenburgische Akademie der Wissenschaften (vormals Preußische Akademie der Wissenschaften) 7/1999, 237-265.

Offe, Claus (2019 [2001]): Wessen Wohl ist das Gemeinwohl? In: Institutionen, Normen, Bürgertugenden. Wiesbaden: Springer, 341-366.

Rink, Dieter / Vollmer, Lisa (2019): „Mietenwahnsinn stoppen!“ Netzwerke und Mobilisierungen der Mieter*innenbewegung in deutschen Großstädten. In: Forschungsjournal Soziale Bewegungen 32/3, 337-349.

Rinn, Moritz (2018): Ein Urbanismus der Ungleichheit. „Neue soziale Stadtpolitik“ in Hamburg als Strategie der Verbürgerlichung. In: sub\urban. zeitschrift für kritische stadtforschung 6/1, 9-28.

Sarnow, Martin (2019): „Wir kaufen den Kiez zurück“. Milieuschutz und Vorkaufsrecht als Ansätze einer postneoliberalen Wohnraumversorgung in Berlin FriedrichshainKreuzberg? In: sub \urban. zeitschrift für kritische stadtforschung 7/1-2, 115-136.

Schipper, Sebastian (2016): Wohnungsnot und Gentrifizierung in Frankfurt am Main. Zur Rolle der städtischen Wohnungsbaugesellschaft ABG Holding in gegenwärtigen Verdrängungsprozessen. https://www.uni-frankfurt.de/45864137/Publikationen (letzter Zugriff am 28.8.2020).

Schipper, Sebastian (2018): Wohnraum dem Markt entziehen? Wohnungspolitik und städtische soziale Bewegungen in Frankfurt und Tel Aviv. Wiesbaden: Springer VS.

Schipper Sebastian / Schönig, Barbara (2021): Die ewig neue Wohnungsfrage! Auf den Spuren bundesdeutscher Debatten zur sozialen Wohnraumversorgung. In: Björn Egner / Stephan Grohs / Tobias Robischon (Hg.), Die Rückkehr der Wohnungsfrage. Ansätze und Herausforderungen lokaler Politik. Wiesbaden: Springer VS, 77-89.

Schönig, Barbara (2018): Ausnahmesegment. Form und Funktion sozialen Wohnungsbausim transformierten Wohlfahrtsstaat. In: PROKLA. Zeitschrift für kritische Sozialwissenschaft 48/191, 227-245.

Schönig, Barbara / Kadi, Justin / Schipper, Sebastian (Hg.) (2017): Wohnraum für alle?! Perspektiven auf Planung, Politik und Architektur. Bielefeld: transcript.

Schönig, Barbara / Vollmer, Lisa (Hg.) (2021): Wohnungsfragen ohne Ende?! Ressourcen für eine soziale Wohnraumversorgung. Bielefeld: transcript.

Senatsverwaltung für Stadtentwicklung und Wohnen (o. J. a): Mieterräte. https:// www.stadtentwicklung.berlin.de/wohnen/wohnraumversorgung/anstalt_themen_ mieterraete.shtml (letzter Zugriff am 17.9.2020). 
Senatsverwaltung für Stadtentwicklung und Wohnen (o. J. b): Wohnraumversorgung Berlin (WVB) - Anstalt öffentlichen Rechts. https://www.stadtentwicklung.berlin.de/wohnen/ wohnraumversorgung/ (letzter Zugriff am 17.9.2020).

Senatsverwaltung für Stadtentwicklung und Wohnen (2020): Stadtentwicklungsplan Wohnen 2030. Neue Wohnungen für Berlin. Berlin.

Stadt Frankfurt (2020): Rechtsgutachten der Stadt Frankfurt. https://www.stadt-fuer-alle. net/?p=1675/ (letzter Zugriff am 7.5.2021).

Stadt für alle (o. J.): Kurzvorstellung Deutsch. https://www.stadt-fuer-alle.net/?page_ id=599 (letzter Zugriff am 5·5.2021).

Stadt für alle (2015): Forderungen Januar 2015. https://www.stadt-fuer-alle.net/?page_ id=59/ (letzter Zugriff am 26.4.2021).

Stadt für alle (2016): Forderungen November 2016. https://www.stadt-fuer-alle.net/?page_ id=1314/ (letzter Zugriff am 26.4.2021).

STVV - Stadtverordnetenversammlung Frankfurt am Main (2014): Wortprotokoll über die 32. Plenarsitzung der Stadtverordnetenversammlung am 26.6.2014. https://www.stvv. frankfurt.de/download/WOP_32_26-06-2014.pdf (letzter Zugriff am 17.9.2020).

Unger, Knut (2018): Mieterhöhungsmaschinen. Zur Finanzialisierung und Industrialisierung der unternehmerischen Wohnungswirtschaft. In: PROKLA. Zeitschrift für kritische Sozialwissenschaft 48/191, 205-225.

Vogelpohl, Anne / Buchholz, Tino (2017): Breaking with neoliberalization by restricting the housing market. Novel urban policies and the case of Hamburg. In: International Journal for Urban and Regional Research 41/2, 266-281.

Vogelpohl, Anne / Vollmer, Lisa / Vittu, Elodie / Brecht, Norma (2017): Die Repolitisierung des Wohnens. Städtische soziale Bewegungen für ein Recht auf Wohnen und auf Stadt in Hamburg, Berlin, Jena und Leipzig. In: Barbara Schönig / Justin Kadi / Sebastian Schipper (Hg.), Wohnraum für alle?! Perspektiven auf Planung, Politik und Architektur. Bielefeld: transcript, 102-131.

Vollmer, Lisa (2019): Mieter_innenbewegungen in Berlin und New York. Die Formierung politischer Kollektivität. Wiesbaden: Springer VS.

Vollmer, Lisa (2021): Gemeinwohlorientierte Stadtentwicklung? Über einen uneindeutigen Begriff und seinen strategischen Einsatz in der stadtpolitischen Bewegung. In: Común. Zeitschrift für stadtpolitische Interventionen, 34-35.

Vollmer, Lisa / Kadi, Justin (2018): Wohnungspolitik in der Krise des Neoliberalismus in Berlin und Wien. Postneoliberaler Paradigmenwechsel oder punktuelle staatliche Beruhigungspolitik? In: PROKLA. Zeitschrift für kritische Sozialwissenschaft 48/191, 247-264.

Yin, Robert K. (2009): Case study research. Design and methods. Thousand Oaks, CA: Sage.

\section{Common good and public housing. Comparing direct democratic initiatives for the realignment of public housing companies in Frankfurt am Main and Berlin}

Rent increases, gentrification and social conflicts are leading to a re-politicization of housing policy, drawing attention to publicly owned housing companies. Social movements increasingly criticize the orientation of municipal and state-owned housing companies questioning the dominant neoliberal orientation of public ownership. In this paper, we position the reform of public housing companies as part of the broader discussion on the common good in housing policy and focus on the challenges of reorganizing publicly owned housing companies. Based on a comparative case study we investigate democratic initiatives in Frankfurt am Main and Berlin that aim to reform public housing companies. In both cities, economic practices of such companies are becoming the object of political movements aiming to profoundly alter these organizations. We document the merits and limits of direct-democratic initiatives and discuss three analytical dimensions of 
the reorganization of public housing companies: Management practices, tenant consultation rights and changes to the legal form of public housing companies. 